\title{
Dapson in heterocyclic chemistry, part VIII: synthesis, molecular docking and anticancer activity of some novel sulfonylbiscompounds carrying biologically active 1,3-dihydropyridine, chromene and chromenopyridine moieties
}

\author{
Mansour S Al-Said ${ }^{1}$, Mostafa M Ghorab ${ }^{1 *}$ and Yassin M Nissan²
}

\begin{abstract}
Several new sulfonebiscompounds having a biologically active 1,2-dihydropyridine-2-one 3-19, acrylamide 20, chromene 21, 22 and chromenopyridine 23, 24 moieties were synthesized and evaluated as potential anticancer agents. The structures of the products were confirmed via elemental analyses and spectral data. The screening tests showed that many of the biscompounds obtained exhibited good anticancer activity against human breast cell line (MCF7) comparable to doxorubicin which was used as reference drug. Compounds 11,17 and 24 showed $I_{50}$ values $35.40 \mu \mathrm{M}, 29.86 \mu \mathrm{M}$ and $30.99 \mu \mathrm{M}$, respectively. In order to elucidate the mechanism of action of the synthesized compounds as anticancer agents, docking on the active site of farnesyltransferase and arginine methyltransferase was also performed and good results were obtained.
\end{abstract}

Keywords: Sulfone, Pyridines, Chromenes, Pyridnochromenes, Anticancer activity

\section{Background}

Many naturally occurring and synthetic compounds containing the 2-pyridone scaffold possess interesting pharmacological properties [1]. The pyridine derivative I, for example, has been identified as specific nonnucleuoside reverse transcriptase inhibitor in treatment of HIV-1 [2,3]. While the pyridine derivatives, Milirinone II and Amrinone III, and their analouges are used as cardiotonic agents in the treatment of heart failure [4-7]. Also, Pirfeidione (PFD) IV, a pyridine derivative which demonstrated antifibrotic activity in several organs in experimental animals, including lung, kidney and uterus has proven beneficial cure for a range of fibrotic conditions through both anti-inflammtory and and antifibrotic mechanisms [8]. A phase II clinical study showed PFD to be promising agent for the treatment of idiopathic pulmonary fibrosis, initiated in mice

\footnotetext{
* Correspondence: mmsghorab@yahoo.com

'Medicinal, Aromatic and Poisonous Plants Research Center (MAPPRC),

College of Pharmacy, King Saud University, 2457, Riyadh 11451, Saudi Arabia Full list of author information is available at the end of the article
}

treated with cyclophosamide [9], amiodarone [10] or belomycin [11-16]. The reported antifbrotic activity of PFD prompted us to synthesize a new series of sulfonebiscompounds carrying biologically active 1,2-dihydropyridine-2one, chromene and chromenopyridine as analoges to PFD. In addition, some 2-pyridones are also reported to possess antitumor, antibacterial [17] and other biological activities [18-20]. On the otherhand, sulfone derivatives have been found to exhibit a wide variety of pharmacological activities [21-25]. In addition, the bisheterocyclic compounds chromenes and chromenopyridine derivatives are well known as anticancer agents [26-29]. Also, diphenylsulfones and bisheterocyclic compounds are reported to have a broad spectrum of biological activities. Some are endowed with antitumor or antifungal properties [30]. On the other hand, some pyridine and isoquinoline derivatives have various biological properties such as antimicrobial [31], anticancer [32-35] activities.

Recent studies have proved the remarkable effect of Dapson on inhibiting cell growth in glioblastoma by acting as anti-VEGF and anti-angiogenic agent via depriving 
glioblastoma of neutrophil-mediated growth promoting effects [36]. Allantodapson V, a Dapson derivative showed high activity as anticancer through inhibition of arginine methyltranseferase (PRMT1) an enzyme which plays an important role in hormone dependent cancers. A series of acylated diarylsulfone derivatives were evaluated for the same activity and compound VI exihibited good activity as (PRMT1) inhibitor [37].

In view of these findings, and in continuation to our work in the synthesis of novel anticancer agents [38-42] we undertook the synthesis of bisheterocyclicsulfone compounds analogues for 2-pyridones incorporating biologically active 1,2-dihydropyridine-2-one, chromene, and chromenopyridone in one molecule to explore the promising anticancer compounds.

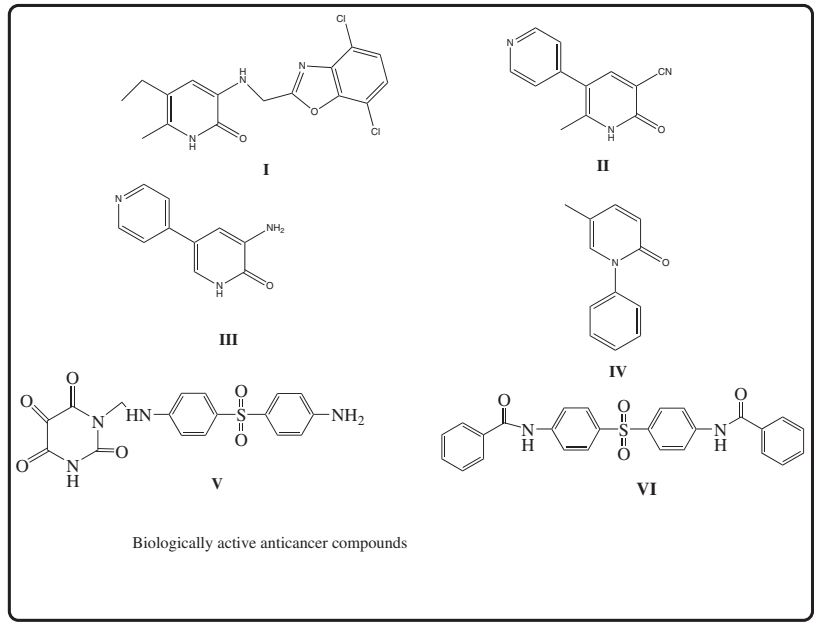

\section{Results and discussion}

\section{Chemistry}

Several compounds were designed with the aim of exploring anticancer properties (Scheme 1, Scheme 2, Scheme 3). Scheme 1 outlines the synthetic pathway used to obtain compounds 3-16. The starting material N,N'-(4,4'-sulfonylbis(4,1-phenylene))bis(2-cyanoacetamid) 2 was obtained via reaction of Dapson 1 with ethyl cyanoacetate. Compound 2 was established by elemental analysis and spectral data. Thus, IR spectrum of 2 revealed bands at 3448, $3363 \mathrm{~cm}^{-1}$ $(2 \mathrm{NH}), 2256 \mathrm{~cm}^{-1}(2 \mathrm{C} \equiv \mathrm{N}), 1701 \mathrm{~cm}^{-1}(2 \mathrm{C}=\mathrm{O})$ and 1342, $1180 \mathrm{~cm}^{-1}\left(\mathrm{SO}_{2}\right) .{ }^{1} \mathrm{H}-\mathrm{NMR}$ spectrum of 2 in (DMSO- $\mathrm{d}_{6}$ ) exhibited signals at $4.0 \mathrm{ppm}$ due to $\mathrm{CH}_{2}$ group, 7.4-7.9 ppm corresponding to aromatic protons and $10.7 \mathrm{ppm}$ due to $2 \mathrm{NH}$ groups. Treatment of compound 2 with appropriate aldehyde and malononitrile in the presence of catalytic amounts of pipredine compounds 3-16, respectively. These compounds were verified on the basis of elemental analyses, IR, ${ }^{1} \mathrm{H}-\mathrm{NMR}$ and ${ }^{13} \mathrm{C}$-NMR. Thus, IR spectra of compounds 3-16 exhibited the presence of $\mathrm{NH}_{2}, \mathrm{C} \equiv \mathrm{N}, \mathrm{C}=\mathrm{O}$ and $\mathrm{SO}_{2}$ bands. ${ }^{1} \mathrm{H}-\mathrm{NMR}$ spectra of compounds $3-16$ in $\left(\mathrm{DMSO}-\mathrm{d}_{6}\right)$ revealed the presence of

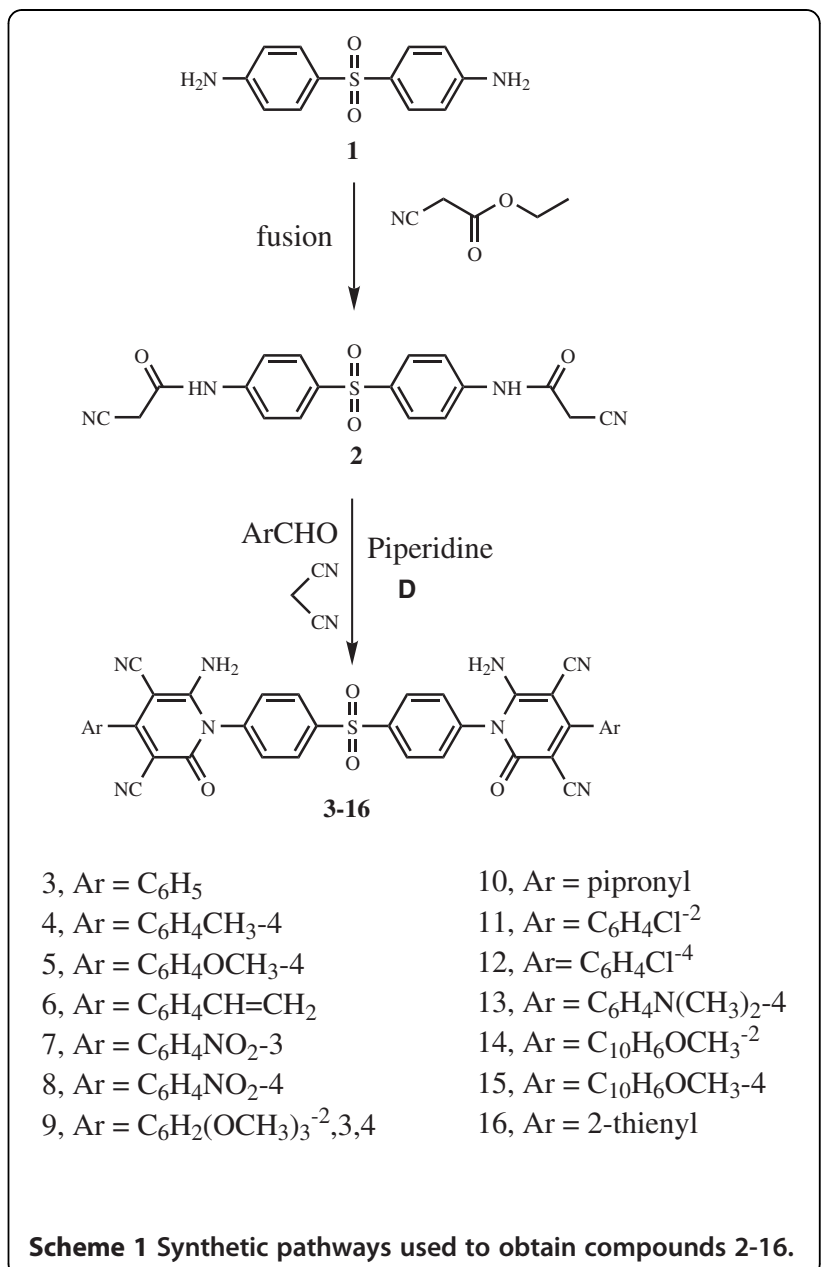

$\mathrm{NH} 2$ at 6.0-6.8 ppm and aromatic protons at 6.9-8.7 ppm (Scheme 1).

Similarly, interaction of 2 with aliphatic aldehyde and malononitrile in ethanol containing catalytic amount of piperidine afforded the corresponding 1,2-dihydropyridine-2-one derivatives 17-19. IR spectra of compounds 17-19, exhibited the presence of characteristic bands of $\mathrm{NH}_{2}, \mathrm{C} \equiv \mathrm{N}, \mathrm{C}=\mathrm{O}$ and $\mathrm{SO}_{2}$ groups ${ }^{1} \mathrm{H}-\mathrm{NMR}$ spectrum of 17 in $\left(\mathrm{DMSO}-\mathrm{d}_{6}\right)$ revealed signals at $6.8 \mathrm{ppm}$ due to $2 \mathrm{NH}_{2}$, while ${ }^{1} \mathrm{H}-\mathrm{NMR}$ spectrum of 18 in (DMSO- $\mathrm{d}_{6}$ ) revealed signals at $1.8 \mathrm{ppm}$ corresponding to $2 \mathrm{CH}_{3}$. On the other hand, ${ }^{1} \mathrm{H}-\mathrm{NMR}$ spectrum of 19 in $\left(\mathrm{DMSO}^{-\mathrm{d}_{6}}\right)$ triplet signal at $1.1 \mathrm{ppm}$ for $\mathrm{CH}_{3}$ and a multiplet one at 1.3-2.0 ppm corresponding to $\mathrm{CH}_{2}$ groups. Interaction of 2 with 2,4-dichlorobenzaldehyde in acetic acid gave the corresponding acrylamide derivative 20 . IR spectrum of 20 revealed bands at $3372 \mathrm{~cm}^{-1}(2 \mathrm{NH}), 2203 \mathrm{~cm}^{-1}(2 \mathrm{C} \equiv \mathrm{N})$, $1652 \mathrm{~cm}^{-1}(2 \mathrm{C}=\mathrm{O})$ and $829 \mathrm{~cm}^{-1}(\mathrm{C}-\mathrm{Cl}) .{ }^{1} \mathrm{H}-\mathrm{NMR}$ spectrum of 20 in (DMSO- $\mathrm{d}_{6}$ ) showed signals at $8.0 \mathrm{ppm}$ due to $\mathrm{CH}$ groups, $10.0 \mathrm{ppm}$ corresponding to $\mathrm{NH}$ groups (Scheme 2). 


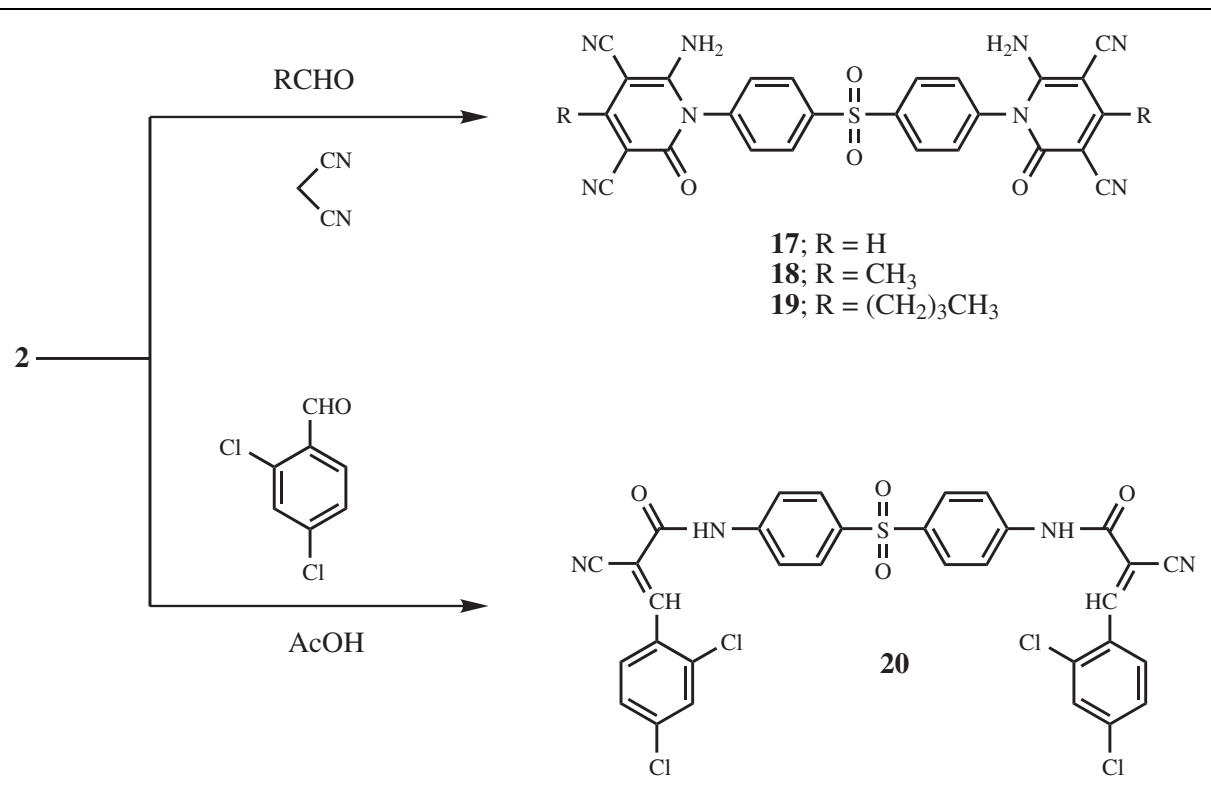

Scheme 2 Synthetic pathways used to obtain compounds 17-20.

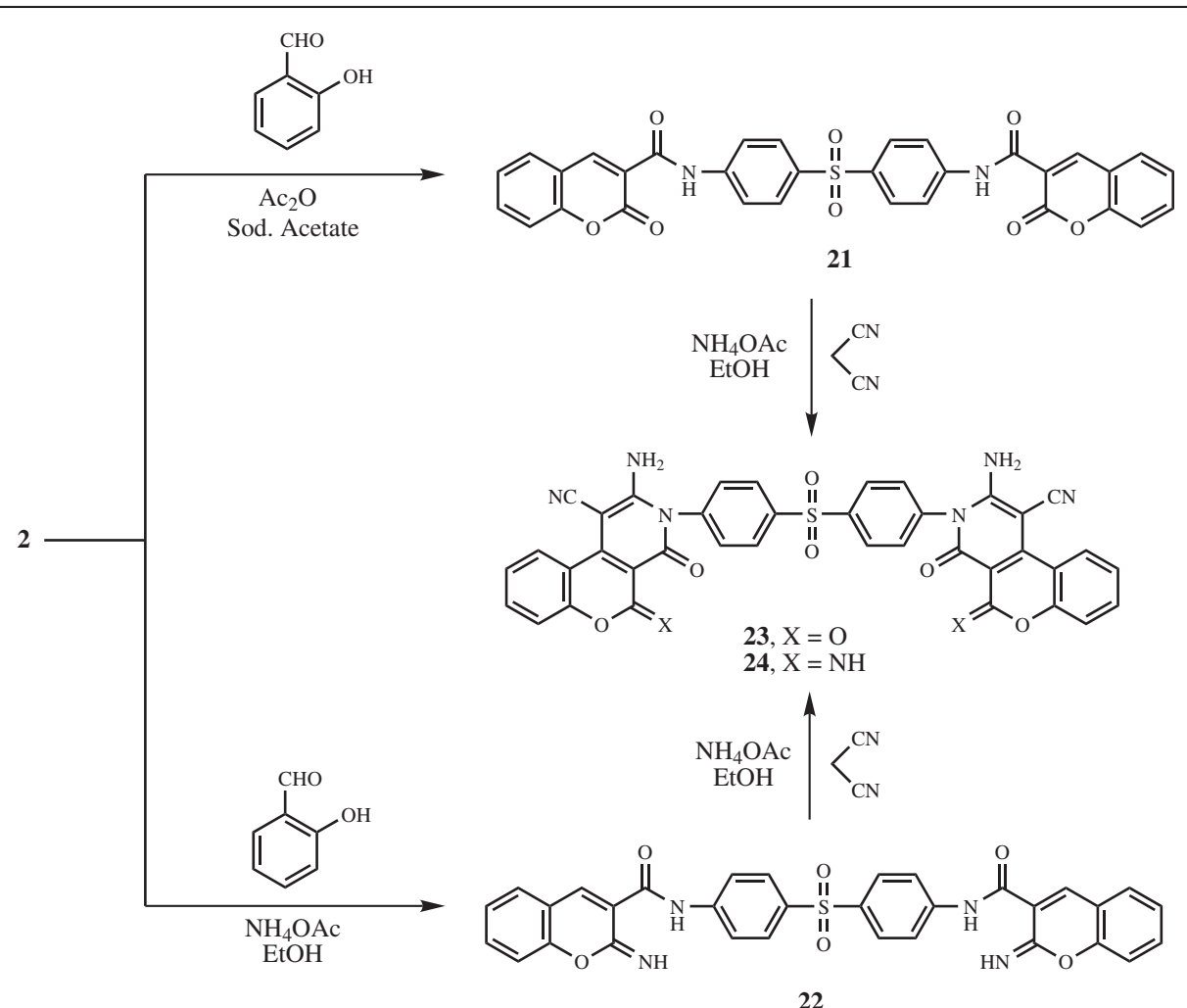

Scheme 3 Synthetic pathways used to obtain compounds 21-24. 
Furthermore, Perkin reaction was carried out by reacting compound 2 with salicylaldehyde in acetic anhydride containing catalytic amount of anhydrous sodium acetate to give the corresponding chromene derivative 21 , while reaction of 2 with salicylaldehyde in ammonium acetate afforded 2-iminochromene derivative 22 (Scheme 3).

\section{Molecular docking}

The zinc-metalloenzyme farnesyl transferase (FTase) catalyzes the transfer of a farnesyl group to a cysteine thiol group contained in the $\mathrm{C}$-terminal tetra peptide signal sequence of Ras, frequently referred to as aCAAX motif. Farnesylation causes membrane localization of Ras which, in turn, determines the switch from an inactive to an active Ras-GTP-bound form [43-45]. Among the Ras isoforms H-ras, N-ras, and K-ras, mutations in the K-ras isoform are most relevant to human cancers in particular pancreatic, colon, and lung cancers, which exhibit approximately 90, 40, and 25\% incidence of Kras mutations, respectively. Inhibitors of FTase prevent membrane localization of the Ras oncogene and have the ability to revert the transformed phenotype, providing the rationale for the development of farnesyl transferase inhibitors (FTIs) as anticancer drugs [46-49].

On the other hand, the relative levels of arginine methyltransferase (PRMT1) isoforms are altered between normal and cancerous breast issue, with two of the isoforms down-regulated [50]. Therefore, it appears that PRMT1expression in cancer cells may be altered depending on the tumor type. Studies are beginning to examine the specific role of PRMT1in cancer. PRMT1 is an essential component of a Mixed Line age Leukaemia (MLL) transcriptional complex that modifies histones by methylation, at H4R3, and acetylation [51]. This serves as the first demonstration of a direct role for PRMT1-mediated transcriptional up regulation during cancer progression.

Thus, the present investigation is concerned with the synthesis of novel anticancer agents and trying to understand their mechanism of action. In order to perform the aim of the present investigations the authors have performed molecular docking of the synthesized compounds on the active sites of both farnesyl transferase and arginine methyltransferase (PRMT1) which may lead to understanding of their effect as antitumor agents.

\section{Molecular docking on the active site of farnesyl transferase}

The protein data bank file (PDB:3E30) was selected for this purpose. The file contains farnesyl transferase enzyme co-crystallized with a sulfone ligand. All docking procedures were achieved by MOE (Molecular Operating Environment) software 10.2008 provided by chemical computing group, Canada. Docking on the active site of farnesyl transferase enzyme was performed for all synthesized compounds 2-24.

Docking protocol was verified by redocking of the cocrystallized ligand in the vicinity of the active site of the enzyme with energy score $(S)=-25.6345 \mathrm{Kcal} / \mathrm{mol}$ and root mean standard deviation $(\mathrm{RMSD})=2.8268$ (Figure 1 ).

The sulfone ligand interacts with the active site of farnesyl transferase by four interactions: Try B361 with a hydrogen bond of $2.95 \mathrm{~A}^{\circ}$ and arene-arene interaction, Trp 102 with a hydrogen bond of 2.83 and with $\mathrm{Zn}$ by the lone pair of imidazole nitrogen. All synthesized compounds were fit to the active site of farnesyl transferase enzyme with good energy scores (S) suggesting activity as farnesyl transferase inhibitors. Energy scores $(\mathrm{S})$ and amino acid interactions for synthesized compounds were listed in (Table 1).

Compound 24 showed the best energy score $(S)=-45.9317$ $\mathrm{Kcal} / \mathrm{mol}$ and interacted with Lys A146 with a hydrogen bond of $2.83 \mathrm{~A}^{\circ}$, with Arg B202 with two hydrogen bonds of $2.45,2.46 \mathrm{~A}^{\circ}$ and with $\mathrm{Zn}$ through its $\mathrm{C}=\mathrm{O}$ and $\mathrm{NH}$ (Figure 2).

\section{Molecular docking on the active site of arginine methyltransferase (PRMT1)}

The protein data bank file (PDB:3Q7E) was selected for this purpose. The file contains arginine methyltransferase co-crystallized with its ligand (S-adenosyl methionine). All docking procedures were achieved by MOE (Molecular Operating Environment)software 10.2008 provided by chemical computing group, Canada. Docking on the active site of arginine methyltransferase enzyme was performed for all synthesized compounds 2-24.

Docking protocol was verified by redocking of the cocrystallized ligand in the vicinity of the active site of the enzyme with energy score $(S)=-18.5932 \mathrm{Kcal} / \mathrm{mol}$ and root mean standard deviation $(\mathrm{RMSD})=0.3523$. The ligand interacts with the active site of arginine methyltransferase by five interactions: Val 128 with a hydrogen bond of $3.00 \mathrm{~A}^{\mathrm{o}}$, with Arg 54 with a hydrogen bond of 2.64, with Gly 78 with a hydrogen bond of $1.81 \mathrm{~A}^{\circ}$ and with Glu 100 with two hydrogen bonds of 181, $186 \mathrm{~A}^{\circ}$ (Figure 3).

All synthesized compounds were fit to the active site of arginine methyltransferase enzyme with good energy scores (S) except compounds 7, 18 and 19 suggesting good activity as arginine methyltransferase inhibitors for most of the synthesized compounds. Energy scores (S) and amino acid interactions for the synthesized compounds were listed in (Table 2).

Compound 24 showed the best energy score $(S)=-23.0582$ $\mathrm{Kcal} / \mathrm{mol}$ and interacted with Arg 327 with two hydrogen bonds of 2.51, $2.46 \mathrm{~A}^{\mathrm{o}}$, with Lys 127 with a hydrogen bond of $2.75 \mathrm{~A}^{\circ}$ and with Glu 130 with a hydrogen bond of $1.36 \mathrm{~A}^{\circ}$ (Figure 4 ). 


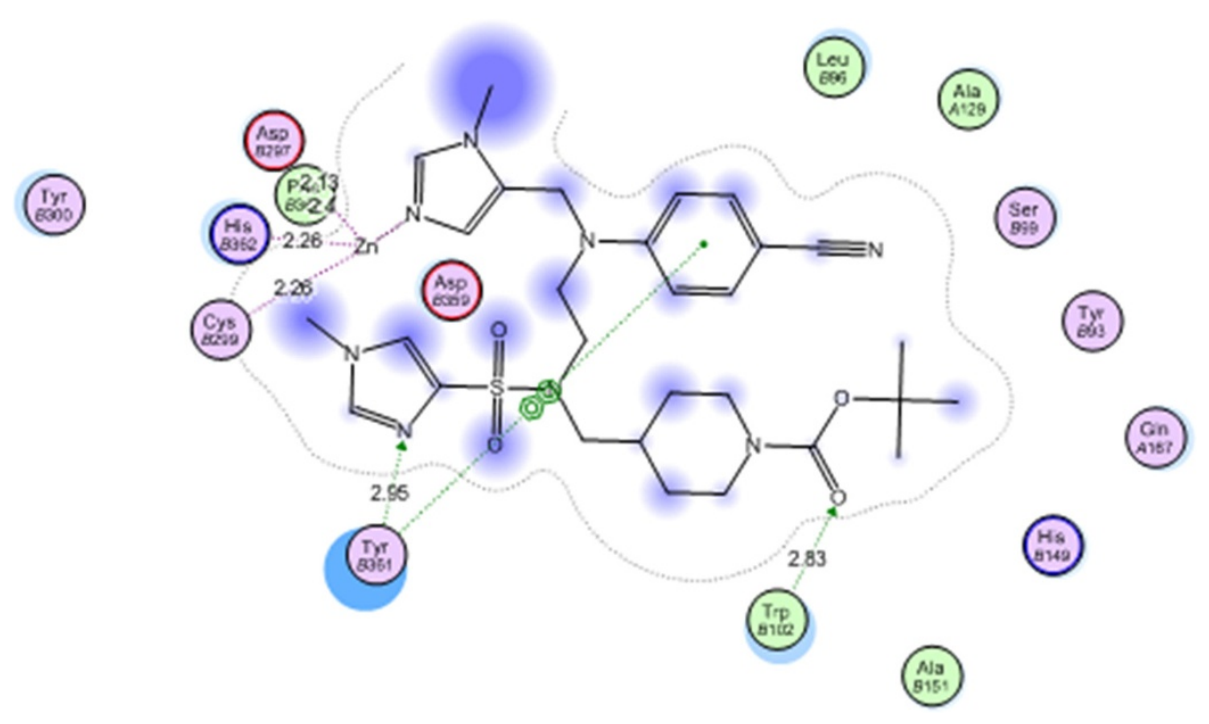

Figure 1 Co-crystallized sulfone ligand on the active site of farnesyltransferase.

Table 1 Binding scores and amino acid interactions of the docked compounds on the active site of farnesyltransferase (FT)

\begin{tabular}{|c|c|c|c|c|}
\hline Compound no. & S Kcal/Mol & Amino acid interactions & $\mathrm{H}$ bond length $\mathrm{A}^{\circ}$ & Interaction with $\mathrm{Zn}$ \\
\hline 2 & -22.2685 & Leu B295, Lys B294 & $3.37,2.76$ & No interaction \\
\hline 3 & -37.4155 & Lys A164, Arg B202 & $3.39,2.53-3.14$ & $\mathrm{CN}$ \\
\hline 4 & -25.1368 & Lys B234, Tyr B334, LysB358, Arg B202 & $3.08,2.75,3.28,2.73$ & $\mathrm{SO}_{2}$ \\
\hline 5 & -22.9916 & Lys B294, Lys A164, Gln A167, Arg B202 & $3.47,2.84,3.00,3.09$ & $\mathrm{C}=\mathrm{O}$ \\
\hline 6 & -31.4218 & Lys A164, Arg B202 & $2.49,3.28$ & $\mathrm{SO}_{2}$ \\
\hline 7 & -30.3616 & Arg B291, Arg B202 & $3.19,2.47-2.96$ & $\mathrm{CN}$ \\
\hline 8 & -26.5141 & Arg B291, Lys B294 & $3.55,2.94$ & $\mathrm{CN}$ \\
\hline 9 & -25.5855 & Lys B294, Lys A168, His B362 & $2.58,2.76,3.19$ & $\mathrm{CN}$ \\
\hline 10 & -27.1374 & Ser B99, Ser B367, Arg B291 & $3.30,3.05,2.56$ & No interaction \\
\hline 11 & -23.4085 & Trp B102, Lys A168 & $2.75,2.80$ & $\mathrm{C}=\mathrm{O}$ \\
\hline 12 & -28.7413 & Lys A164, Ser B99 & $3.00,3.25$ & $\mathrm{CN}$ \\
\hline 13 & -27.1676 & Lys A164, Arg B202 & $2.48,2.76$ & $\mathrm{SO}_{2}$ \\
\hline 14 & -28.8232 & Lys A164, Arg B202 & $2.81,2.89-3.25$ & $\mathrm{C}=\mathrm{O}$ \\
\hline 15 & -32.2519 & Tyr B300, Asn A165 & $3.10,3.32$ & $\mathrm{CN}$ \\
\hline 16 & -38.0536 & Arg B202, Arg B291, Lys B294 & $2.57,3.01,3.39$ & $\mathrm{CN}$ \\
\hline 17 & -19.9521 & Lys B353, Gly B290, Lys B294, Arg B202 & $2.78,3.29,2.67,3.13$ & No interaction \\
\hline 18 & -23.0290 & Leu B295, Lys B294 & $3.05,2.61$ & No interaction \\
\hline 19 & -32.9232 & Arg B291 & 3.81 & $\mathrm{CN}$ \\
\hline 20 & -24.4073 & Arg B202 & 2.35 & $\mathrm{C}=\mathrm{O}$ \\
\hline 21 & -29.7807 & Tyr B300 & 2.85 & $\mathrm{C}=\mathrm{O}$ \\
\hline 22 & -38.6191 & Arg B202, Asp B352 & $2.92,1.96$ & $\mathrm{C}=\mathrm{O}, \mathrm{NH}$ \\
\hline 23 & -38.8898 & Lys A164, Arg B202 & $2.81,2.49-2.55$ & $C=O, C=O$ \\
\hline 24 & -45.9317 & Lys A164, Arg B202 & $2.83,2.46-2.45$ & $\mathrm{C}=\mathrm{O}, \mathrm{NH}$ \\
\hline
\end{tabular}




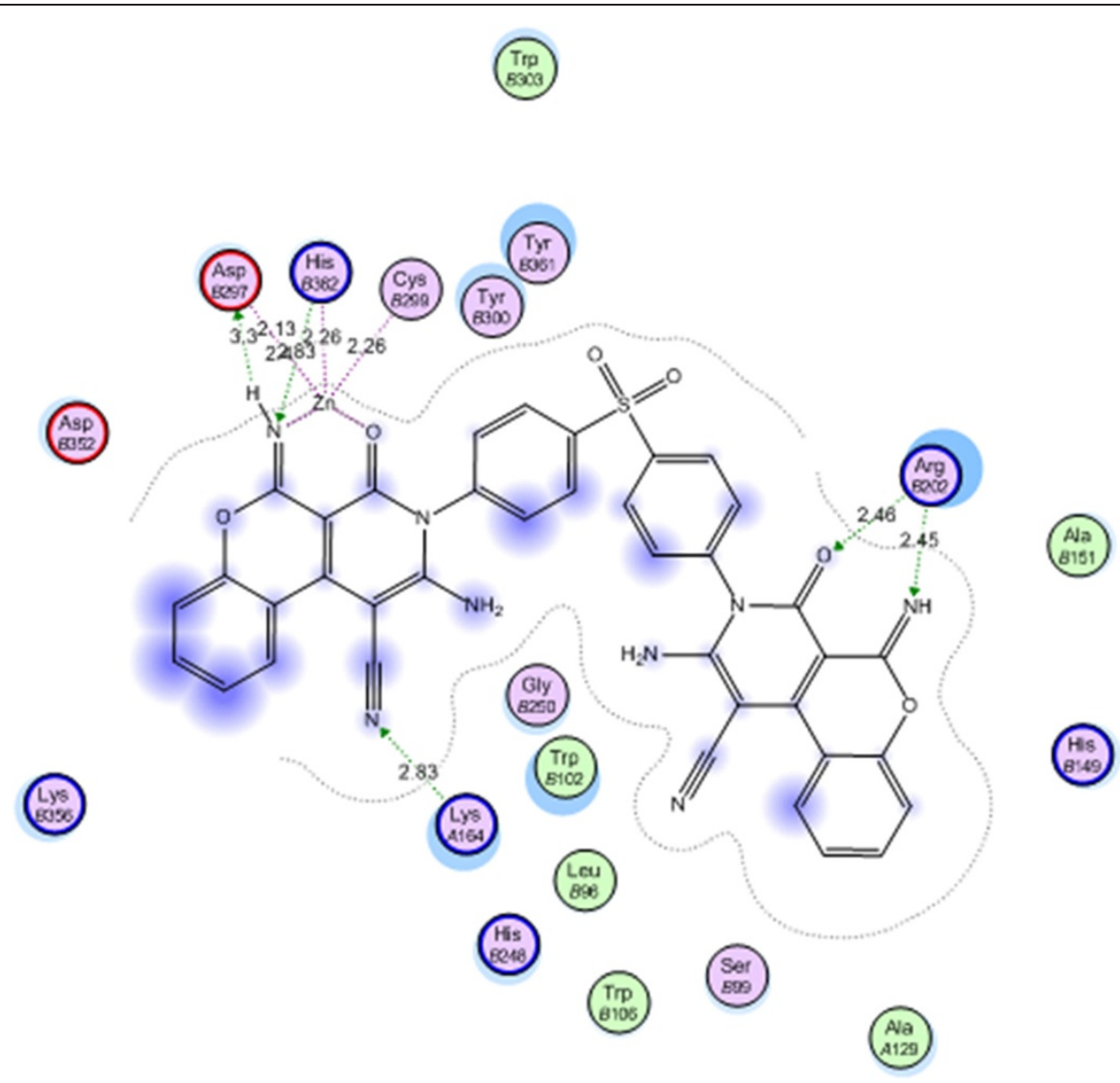

Figure $\mathbf{2}$ Compound 24 on the active site of farnesyltransferase.

\section{In vitro antitumor activity}

The newly synthesized compounds were evaluated for their in vitro cytotoxic activity against human breast cancer cell line; MCF7. Doxorubicin which is one of the most effective anticancer agents was used as the reference drug in this study. The relationship between surviving fraction and drug concentration was plotted to obtain the survival curve of breast cancer cell line (MCF7). The response parameter calculated was the $\mathrm{IC}_{50}$ value, which corresponds to the concentration required for $50 \%$ inhibition of cell viability. Table 3 shows the in vitro cytotoxic activity of the synthesized compounds where all compounds exhibited significant activity compared to the reference drug.

All the synthesized compounds showed better cytotoxic activity than Doxorubicin except compound 5 which showed $\mathrm{IC}_{50}$ value $81.22 \mu \mathrm{M}$. The 1,2-dihdropyridine-2-one derivatives 3-19 showed $\mathrm{IC}_{50}$ values in the rang 29.86$81.22 \mu \mathrm{M}$. Compound 17 which showed $\mathrm{IC}_{50}$ value $29.86 \mu \mathrm{M}$ was the most active compound. Compound 17 also showed good scoring energy $\mathrm{S}=-19.9521 \mathrm{kcal} / \mathrm{Mol}$. and the good amino acid interactions upon docking on the active site of farnesyl transferase enzyme. It also showed good energy score $\mathrm{S}=-20.9464 \mathrm{kcal} / \mathrm{Mol}$. and good amino acid interactions upon docking on the active site of arginine methyl transferase enzyme. Upon substitution on position 4 of compound 17 with several substitutions the activity drops. However, 2,3,4-trimethoxy phenyl substitution, 2-chloro phenyl substitution and 4-chloro phenyl substitution did not decrease the activity in the same way substitution with 4- $\mathrm{CH}_{3}$ phenyl, 4- $\mathrm{OCH}_{3}$ phenyl and $2-\mathrm{OCH}_{3}$ naphthyl did. This was clearly illustrated by the values of $\mathrm{IC}_{50}$ of the 1,2-dihdropyridine-2-one derivatives 9, 11 and 12 with $\mathrm{IC}_{50}$ values of $37.29 \mu \mathrm{M}, 35.40 \mu \mathrm{M}$ and 40.12 respectively. On the other hand, the $\mathrm{IC}_{50}$ values for the 1,2 dihdropyridine-2-one derivatives in which the substitution was with 4- $\mathrm{CH}_{3}$ phenyl, 4- $\mathrm{OCH}_{3}$ phenyl and $2-\mathrm{OCH}_{3}$ naphthyl were much higher indicating less activity. This was clearly shown in the 1,2-dihydropyridine derivatives 4,5 and 14 with $\mathrm{IC}_{50}$ values of $54.37 \mu \mathrm{M}, 81.22 \mu \mathrm{M}$ and $65.58 \mu \mathrm{M}$, respectively.

Compounds 20-24 showed cytotoxic activity with $\mathrm{IC}_{50}$ values in the range of 30.99 to $56.37 \mu \mathrm{M}$ with cytotoxic activity better than that of Doxorubicin. The chromenopyridine derivative 24 was with the best $\mathrm{IC}_{50}=30.99 \mu \mathrm{M}$ among these compounds while compound 23 showed the highest $\mathrm{IC}_{50}$ value $56.37 \mu \mathrm{M}$ among these compounds. 


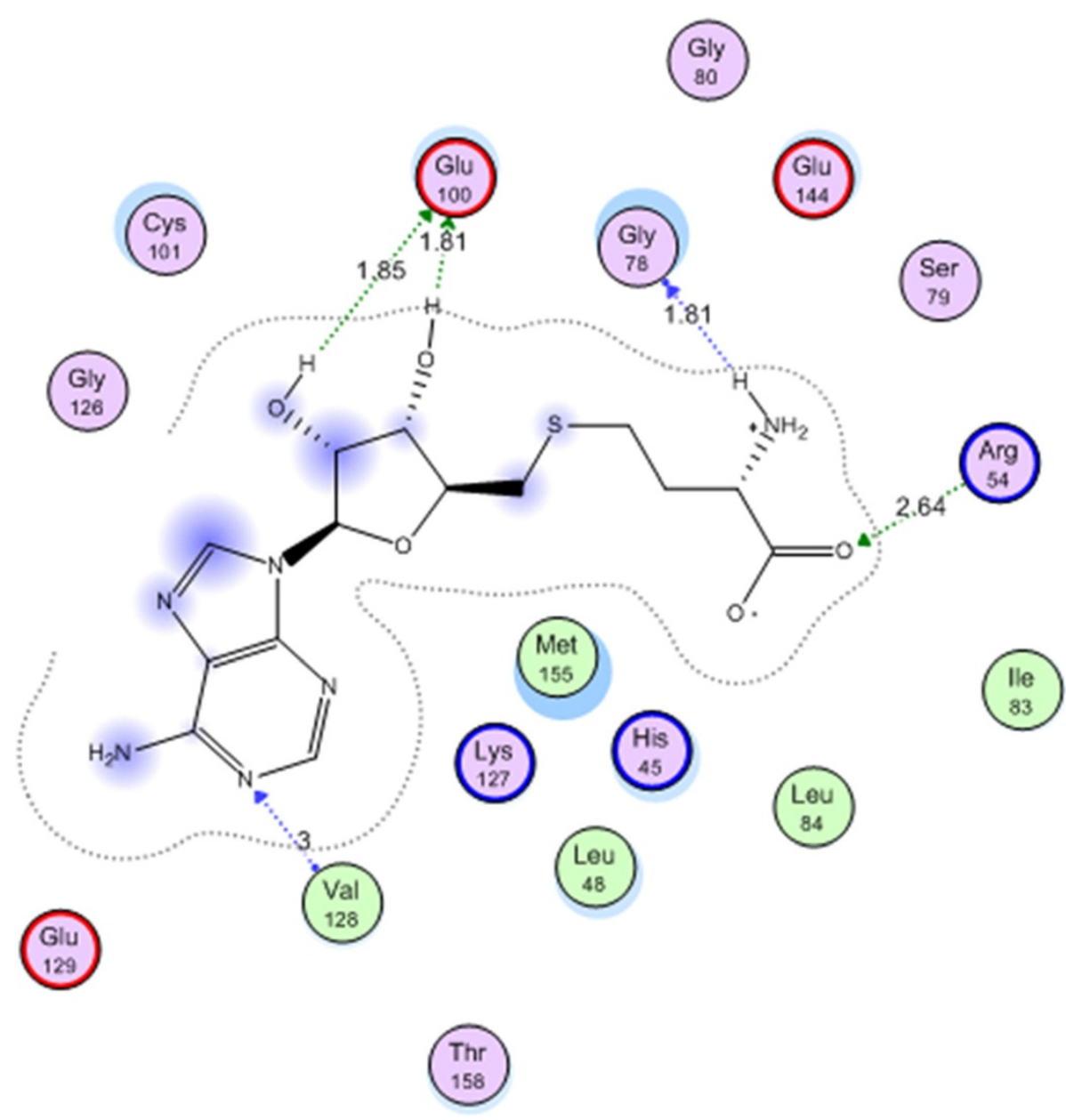

Figure 3 Co-crystallized S-adenosyl methionine ligand on the active site of arginine methyltransferase (PRMT1).

Compound 24 also showed the best scoring energy $\mathrm{S}=-45.9317 \mathrm{kcal} / \mathrm{Mol}$. and the best amino acid interactions upon docking on the active site of farnesyl transferase enzyme. It also showed the best energy score $\mathrm{S}=-23.0582 \mathrm{kcal} / \mathrm{Mol}$. and the best amino acid interactions upon docking on the active site of arginine methyl transferase enzyme.

The promising results of cytotoxic activity of the synthesized compounds especially compounds 17, 24 urge more investigations for their mechanism of action. The trial in the present investigation to predict an assumption of the mechanism of action of the synthesized compounds was conducted through molecular docking on the active site of two enzymes based on the similarities between the synthesized compounds and the enzyme inhibitors of these enzymes.

\section{Experimental}

\section{Chemistry}

Melting points $\left({ }^{\circ} \mathrm{C}\right.$, uncorrected) were determined in open capillaries on a Gallenkemp melting point apparatus (Sanyo
Gallenkemp, Southborough, UK) and were uncorrected. Precoated silica gel plates (silica gel 0.25 mm, 60 GF254; Merck, Germany) were used for thin layer chromatography, dichloromethane/methanol (9.5:0.5) mixture was used as a developing solvent system and the spots were visualized by ultraviolet light and/or iodine. Infra-red spectra were recorded in $\mathrm{KBr}$ discs using IR-470 Shimadzu spectrometer (Shimadzu, Tokyo, Japan). NMR spectra (in DMSO-d6) were recorded on Bruker AC-300 Ultra Shield NMR spectrometer (Bruker, Flawil, Switzerland, $\delta$ ppm) at $300 \mathrm{MHz}$ using TMS as internal Standard and peak multiplicities are designed as follows: s, singlet; $\mathrm{d}$, doublet; $\mathrm{t}$, triplet; $\mathrm{m}$, multiplet. Elemental analyses were performed on Carlo Erba 1108 Elemental Analyzer (Heraeus, Hanau, Germany).

N,N'-(4,4'-sulfonylbis(4,1-phenylenebis (2-cyanoacetamide) 2 A mixture of Dapsone (2.48 g, $0.01 \mathrm{~mol}$.) and ethyl cyanoacetate (1.13 g, $0.01 \mathrm{~mol}$.) was refluxed for $3 \mathrm{~h}$, concentrated and cooled. The obtained solid was 
Table 2 Binding scores and amino acid interactions of the docked compounds on the active site of arginine methyltransferase (PRMT1)

\begin{tabular}{|c|c|c|c|}
\hline Compound no. & S Kcal/Mol & Amino acid interactions & $\mathrm{H}$ bond length $\mathrm{A}^{\circ}$ \\
\hline 2 & -20.0584 & Lys 127, His 293 & $2.65,2.81$ \\
\hline 3 & -13.8464 & Lys 127, Arg 327 & $2.39,2.96$ \\
\hline 4 & -17.2063 & Lys 127, Arg 327 & $2.42-2.39,2.45$ \\
\hline 5 & -13.6909 & Lys 127, His 45, Arg 327 & $2.57,2.95,2.36$ \\
\hline 6 & -18.0294 & Arg 327 & $2.45-3.02$ \\
\hline 7 & 11.0959 & -—-— & \\
\hline 8 & -15.9006 & Lys 127, Arg 327 & $2.40,2.30$ \\
\hline 9 & -5.1052 & His 45, Glu 153, Arg 327 & $2.75,1.65,2.36$ \\
\hline 10 & -17.1347 & Lys 127, Glu 153, His 45 & $2.75,1.58,2.87$ \\
\hline 11 & -12.0837 & Asn 167 & 2.65 \\
\hline 12 & -19.6261 & Lys 127, Arg 327 & $2.59-2.84,2.85$ \\
\hline 13 & -15.7402 & Lys 127, Glu 153, Arg 327 & $2.47,1.93,2.44$ \\
\hline 14 & -20.4078 & Asn 157, Lys 127 & $3.18,2.66-2.79$ \\
\hline 15 & -18.8629 & Gln 163, Lys 127 & $2.22,2.42-3.23$ \\
\hline 16 & 14.8212 & -二--二-—- & -———— \\
\hline 17 & -20.6494 & Asn 157, His 45, Lys 127 & $3.24,3.21,2.68$ \\
\hline 18 & 6.1835 & --ー-ー-ー- - & - \\
\hline 19 & 10.1989 & -——-—-— & - \\
\hline 20 & -17.2838 & Lys 127 & 2.51 \\
\hline 21 & -17.6535 & Lys 127 & $2.51,2.86$ \\
\hline 22 & -15.4395 & Arg 327, Glu 144 & $2.79,1.47$ \\
\hline 23 & -19.4615 & Lys 127 & $2.54,2.52$ \\
\hline 24 & -23.0582 & Arg 327, Lys 127, Glu 130 & $2.51-2.46,2.75,1.36$ \\
\hline
\end{tabular}

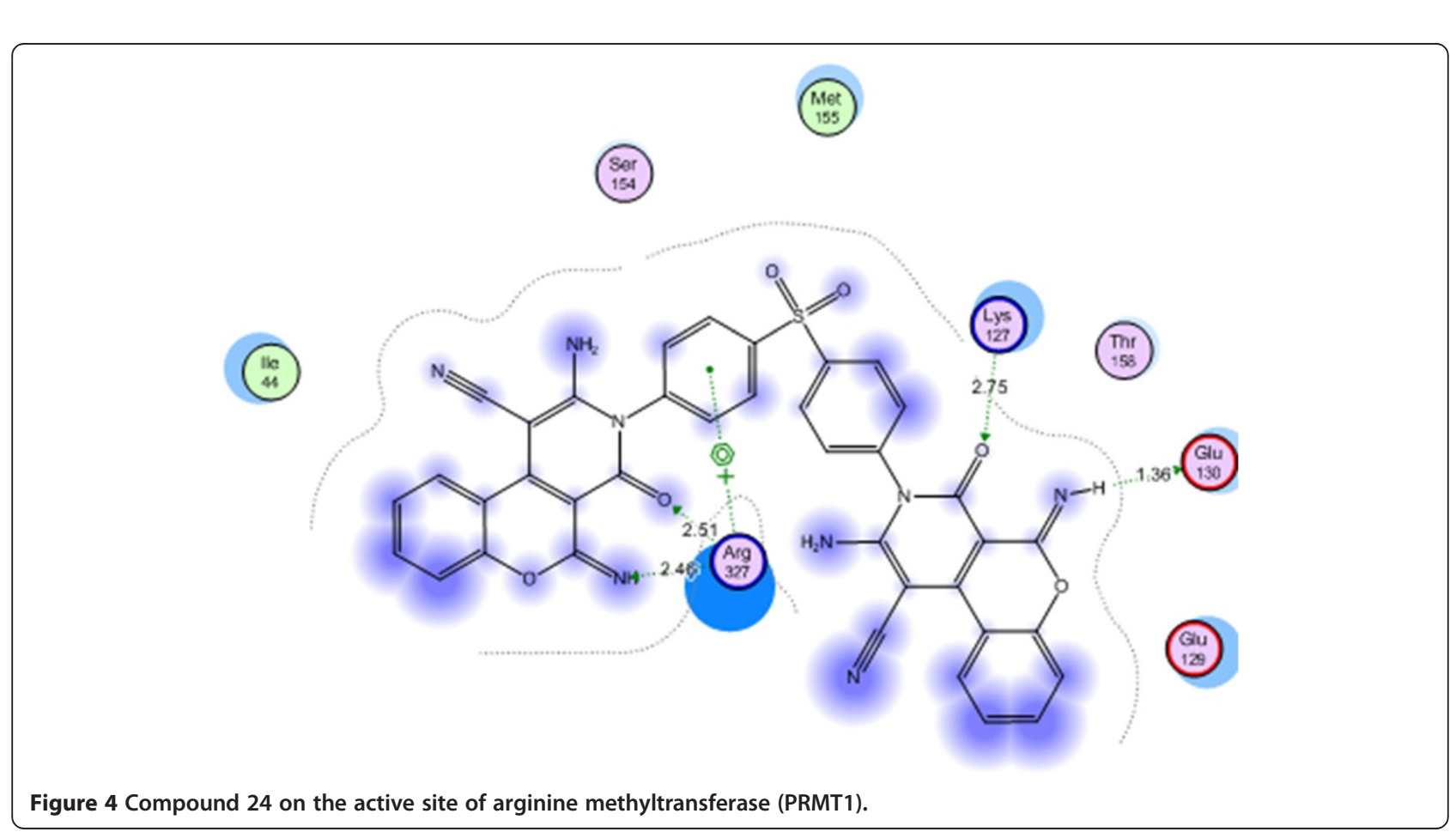


Table 3 In vitro anticancer screening of the synthesized compounds against human breast cell line (MCF7)

\begin{tabular}{|c|c|c|c|c|}
\hline \multirow[t]{2}{*}{ Comp NO. } & \multicolumn{4}{|c|}{ Compound concentration $(\mu \mathrm{M})$} \\
\hline & $10 \mu \mathrm{M}$ & $25 \mu \mathrm{M}$ & $50 \mu \mathrm{M}$ & $100 \mu \mathrm{M}$ \\
\hline & Surviv & 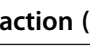 & \pm S.E.) $)^{*}$ & \\
\hline
\end{tabular}

\begin{tabular}{lllll}
\hline Doxorubicin $0.721 \pm 0.02$ & $0.546 \pm 0.02$ & $0.461 \pm 0.01$ & $0.494 \pm 0.03$ & 71.80
\end{tabular}

$2 \quad 0.727 \pm 0.1340 .427 \pm 0.0550 .307 \pm 0.0290 .317 \pm 0.02146 .57$

$3 \quad 0.793 \pm 0.0550 .454 \pm 0.097 \quad 0.292 \pm 0.0080 .332 \pm 0.05052 .45$

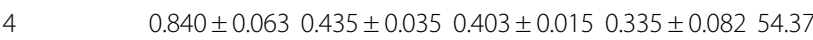

$5 \quad 0.906 \pm 0.0210 .642 \pm 0.0590 .428 \pm 0.038 \quad 0.547 \pm 0.04681 .22$

$6 \quad 0.732 \pm 0.3330 .584 \pm 0.0460 .406 \pm 0.0690 .229 \pm 0.09749 .65$

$7 \quad 0.761 \pm 0.1900 .546 \pm 0.123 \quad 0.254 \pm 0.0310 .297 \pm 0.04847 .83$

$8 \quad 0.830 \pm 0.1240 .399 \pm 0.0820 .199 \pm 0.0210 .272 \pm 0.00542 .56$

$9 \quad 0.649 \pm 0.0280 .394 \pm 0.3390 .207 \pm 0.0270 .261 \pm 0.04937 .29$

$10 \quad 0.609 \pm 0.0590 .479 \pm 0.0950 .332 \pm 0.0580 .316 \pm 0.06445 .45$

$11 \quad 0.747 \pm 0.197 \quad 0.359 \pm 0.0520 .153 \pm 0.0200 .189 \pm 0.00235 .40$

$12 \quad 0.604 \pm 0.0750 .232 \pm 0.0190 .376 \pm 0.0890 .312 \pm 0.02940 .12$

$13 \quad 0.650 \pm 0.1840 .401 \pm 0.0160 .253 \pm 0.0210 .401 \pm 0.01745 .77$

$14 \quad 0.875 \pm 0.0660 .580 \pm 0.0460 .336 \pm 0.0490 .467 \pm 0.04765 .58$

$15 \quad 0.886 \pm 0.0470 .423 \pm 0.0240 .259 \pm 0.054 \quad 0.389 \pm 0.04752 .48$

$16 \quad 0.669 \pm 0.1140 .539 \pm 0.0880 .276 \pm 0.0640 .259 \pm 0.08044 .62$

$17 \quad 0.509 \pm 0.2350 .230 \pm 0.1390 .300 \pm 0.1340 .279 \pm 0.06529 .86$

$18 \quad 0.865 \pm 0.0570 .615 \pm 0.0480 .232 \pm 0.0460 .286 \pm 0.07150 .74$

$19 \quad 0.815 \pm 0.0420 .545 \pm 0.1090 .264 \pm 0.0440 .336 \pm 0.09651 .48$

$20 \quad 0.703 \pm 0.1890 .427 \pm 0.1940 .251 \pm 0.0260 .374 \pm 0.08546 .26$

$21 \quad 0.941 \pm 0.0200 .472 \pm 0.2090 .199 \pm 0.090 \quad 0.278 \pm 0.10847 .49$

$22 \quad 0.653 \pm 0.2910 .574 \pm 0.1800 .337 \pm 0.1160 .359 \pm 0.04452 .74$

$23 \quad 0.878 \pm 0.0320 .563 \pm 0.065 \quad 0.276 \pm 0.031 \quad 0.389 \pm 0.05856 .37$

$24 \quad 0.648 \pm 0.3290 .280 \pm 0.154 \quad 0.174 \pm 0.105 \quad 0.194 \pm 0.06530 .99$

* Each value is the mean of three values \pm Standard Error.

filtered and crystallized from ethanol to give 2. Yield $92 \%$, melting point $137.5-139^{\circ} \mathrm{C}$. IR: $v_{\max } \cdot / \mathrm{cm}^{-1} 3448,3363(2$ $\mathrm{NH}), 3062$ (CH aromatic), 2960, 2931 (CH aliphatic), 2256 (CN), $1701(2 \mathrm{C}=\mathrm{O}), 1342,1180\left(\mathrm{SO}_{2}\right) .{ }^{1} \mathrm{H}-\mathrm{NMR}$ (DMSO$\left.\mathrm{d}_{6}, \mathrm{D}_{2} \mathrm{O}\right): \delta 4.0\left(\mathrm{~s}, 4 \mathrm{H}, 2 \mathrm{CH}_{2}\right), 7.4-7.9(\mathrm{~m}, 8 \mathrm{H}, \mathrm{Ar}-\mathrm{H}), 10.7$ (s, $2 \mathrm{H}, 2 \mathrm{NH}$ exch.). ${ }^{13} \mathrm{C}-\mathrm{NMR}\left(\mathrm{DMSO}-\mathrm{d}_{6}, \mathrm{D}_{2} \mathrm{O}\right): 24.4(2)$, 115.6(2), 119.2(2), 119.3(2), 128.1(2), 129.2(2), 137.8(2), 142.7(2), 162.2(2). Anal. Calcd. for $\mathrm{C}_{18} \mathrm{H}_{14} \mathrm{~N}_{4} \mathrm{O}_{4} \mathrm{~S}$ (382.39): C, 56.54; H, 3.69; N, 14.65. Found: C, 56.81; H, 3.84; N, 14.29.

\section{General procedure for compounds 3-16 and 17-19}

A mixture of the starting material $2(6.86 \mathrm{~g}, 0.01 \mathrm{~mol}$.), appropriate aldehydes $(0.01 \mathrm{~mol}$.) and malononitrile $(0.66 \mathrm{~g}, 0.01 \mathrm{~mol}$.) in ethanol $(50 \mathrm{~mL})$ containing catalytic amount of piperidine in ethanol $(50 \mathrm{~mL})$ was heated under reflux for $5 \mathrm{~h}$. The obtained solid was crystallized from dioxane to give $3-19$, respectively. 1,1'-(4,4'-sulfonylbis(4,1-phenylene))bis(6-amino-2oxo-4-phenyl-1,2-dihydropyridine-3,5-dicarbonitrile) 3 Yield $68 \%$, melting point $257.7-259^{\circ} \mathrm{C}$. IR: $: v_{\max } \cdot / \mathrm{cm}^{-1}$ 3448, $3371\left(2 \mathrm{NH}_{2}\right), 3077(\mathrm{CH}$ aromatic), $2210(2 \mathrm{C} \equiv \mathrm{N})$, $1670(2 \mathrm{C}=\mathrm{O}), 1290(2 \mathrm{C}=\mathrm{S}), 1399,1149\left(\mathrm{SO}_{2}\right) .{ }^{1} \mathrm{H}-\mathrm{NMR}$ $\left(\right.$ DMSO $\left.\mathrm{d}_{6}, \mathrm{D}_{2} \mathrm{O}\right): \delta 6.2\left(\mathrm{~s}, 4 \mathrm{H}, 2 \mathrm{NH}_{2}\right.$, exch.), 7.4-7.9 (m, $18 \mathrm{H}$, Ar-H). Anal. Calcd. for $\mathrm{C}_{38} \mathrm{H}_{22} \mathrm{~N}_{8} \mathrm{O}_{4} \mathrm{~S}(686.70)$ : C, 66.46; H, 3.23; N, 16.32. Found: C, 66.71; H, 3.20; N, 16.00.

\section{1,1'-(4,4'-sulfonylbis(4,1-phenylene))bis(6-amino-2-} oxo-4-p-tolyl-1,2-dihydropyridine-3,5-dicarbonitrile) 4 Yield $76 \%$, melting point $234.2^{\circ} \mathrm{C}$. IR: $\mathrm{v}_{\max } / \mathrm{cm}^{-1} 3371$, $3209\left(2 \mathrm{NH}_{2}\right), 3100(\mathrm{CH}$ aromatic), 2960, $2870(\mathrm{CH}$ aliphatic), $2218(2 \mathrm{C} \equiv \mathrm{N}), 1674 \quad(2 \mathrm{C}=\mathrm{O}), 1400,1149$ $\left(\mathrm{SO}_{2}\right) .{ }^{1} \mathrm{H}$-NMR (DMSO-d $\left.\mathrm{d}_{6}, \mathrm{D}_{2} \mathrm{O}\right): \delta 2.4\left(\mathrm{~s}, 6 \mathrm{H}, 2 \mathrm{CH}_{3}\right)$, $6.6\left(\mathrm{~s}, 4 \mathrm{H}, 2 \mathrm{NH}_{2}\right.$, exch.), 7.3-8.1 (m, $\left.16 \mathrm{H}, \mathrm{Ar}-\mathrm{H}\right) .{ }^{13} \mathrm{C}-$ NMR(DMSO-d $\mathrm{d}_{6}, \mathrm{D}_{2} \mathrm{O}$ ): 17.6 (2), 80.2 (2), 114.2 (2), 115.6 (4), 128.0 (4), 128.6 (4), 130.1 (4), 131.0 (4), 133.8 (4), 134.7 (4), 152.8 (2), 153.9 (2), 163.4 (2). Anal. Calcd. for$\mathrm{C}_{40} \mathrm{H}_{26} \mathrm{~N}_{8} \mathrm{O}_{4} \mathrm{~S}(714.75)$ : C, 67.22; $\mathrm{H}, 3.67 ; \mathrm{N}, 15.68$. Found: C, 67.56; H, 3.44; N, 15.50 .

1,1'-(4,4'-sulfonylbis(4,1-phenylene))bis(6-amino-4(4-methoxyphenyl)-2-oxo-1,2-dihydropyridine-3,5dicarbonitrile) 5 Yield $71 \%$, melting point $>340^{\circ} \mathrm{C}$. IR: $v_{\max } / \mathrm{cm}^{-1} 3390,3210\left(\mathrm{NH}, \mathrm{NH}_{2}\right), 3100(\mathrm{CH}$ aromatic), 2962, 2839 ( $\mathrm{CH}$ aliphatic), 2214 $(2 \mathrm{C} \equiv \mathrm{N}), 1680$ $(2 \mathrm{C}=\mathrm{O}), 1400,1180 \quad\left(\mathrm{SO}_{2}\right) .{ }^{1} \mathrm{H}-\mathrm{NMR} \quad\left(\mathrm{DMSO}-\mathrm{d}_{6}\right.$, $\left.\mathrm{D}_{2} \mathrm{O}\right): \delta 3.8\left(\mathrm{~s}, 6 \mathrm{H}, 2 \mathrm{OCH}_{3}\right), 6.3\left(\mathrm{~s}, 4 \mathrm{H}, 2 \mathrm{NH}_{2}\right.$, exch.), 7.0-8.1 (m, $16 \mathrm{H}$, Ar-H). ${ }^{13} \mathrm{C}-\mathrm{NMR}\left(\mathrm{DMSO}_{\mathrm{d}}, \mathrm{d}_{6} \mathrm{O}\right)$ : 55.2 (2), 77.9 (2), 113.7 (4), 117.6 (2), 117.9 (4), 127.5 (4), 129.1 (2), 129.5 (4), 130.8 (4), 140.6 (2), 141.2 (2), 157.7 (2), 159.6 (2), 160.3 (2), 160.8 (2). Anal. Calcd. for$\mathrm{C}_{40} \mathrm{H}_{26} \mathrm{~N}_{8} \mathrm{O}_{6} \mathrm{~S}(746.75): C, 64.34 ; \mathrm{H}, 3.51 ; \mathrm{N}, 15.01$. Found: C, 64.24; H, 3.19; N, 15.38 .

1,1'-(4,4'-sulfonylbis(4,1-phenylene))bis(6-amino-2-oxo4-styryl-1,2-dihydropyridine-3,5-dicarbonitrile) 6 Yield $79 \%$, melting point $235.8^{\circ} \mathrm{C}$. IR: $v_{\max } \cdot / \mathrm{cm}^{-1} 3448,3370$ $\left(2 \mathrm{NH}_{2}\right), 2939,2860$ ( $\mathrm{CH}$ aliph.), $2218(2 \mathrm{C} \equiv \mathrm{N}), 1685$ $(2 \mathrm{C}=\mathrm{O}), 1390,1149 \quad\left(\mathrm{SO}_{2}\right) .{ }^{1} \mathrm{H}-\mathrm{NMR} \quad\left(\mathrm{DMSO}_{\mathrm{d}}\right.$, $\left.\mathrm{D}_{2} \mathrm{O}\right): \delta 6.6,6.9(2 \mathrm{~d}, 4 \mathrm{H}, 2 \mathrm{CH}=\mathrm{CH}, \mathrm{J}=7.4,7.3 \mathrm{~Hz})$, 7.0-7.8 (m, $22 \mathrm{H}, \mathrm{Ar}-\mathrm{H}+2 \mathrm{NH}_{2}$, exchangable). ${ }^{13} \mathrm{C}-\mathrm{NMR}$ (DMSO-d $\left.\mathrm{d}_{6}\right)$ : 62.9(2), 101.8(2), 113.0(4), 120.7(4), 125.7 (2), 126.8(4), 128.1(2), 128.5(4), 129(4), 131.9(2), 133.7 (2), 137.2(2), 138.8(2), 154.3(2), 155.9(2), 176.1(2). Anal. Calcd. for $\mathrm{C}_{42} \mathrm{H}_{26} \mathrm{~N}_{8} \mathrm{O}_{4} \mathrm{~S}(738.77)$ : C, 68.28; $\mathrm{H}$, 3.55; N, 15.17. Found: C, 68.53; H, 3.61; N, 14.92.

$1,1^{\prime}$-(4,4'-sulfonylbis(4,1-phenylene))bis(6-amino-4(3-nitrophenyl)-2-oxo-1,2-dihydropyridine-3,5-dicarbonitrile) 7 Yield $81 \%$, melting point $167.2^{\circ} \mathrm{C}$. IR: $: v_{\max } \cdot / \mathrm{cm}^{-1}$ 3375, $3213\left(2 \mathrm{NH}_{2}\right), 3093(\mathrm{CH}$ arom.), $2218(2 \mathrm{C} \equiv \mathrm{N})$, 1674 $(2 \mathrm{C}=\mathrm{O}), 1350,1149\left(\mathrm{SO}_{2}\right), 1593,1330\left(\mathrm{NO}_{2}\right)$. 
${ }^{1} \mathrm{H}-\mathrm{NMR} \quad\left(\mathrm{DMSO}-\mathrm{d}_{6}, \quad \mathrm{D}_{2} \mathrm{O}\right): \delta \quad 6.6 \quad\left(\mathrm{~s}, \quad 4 \quad \mathrm{H}, \quad 2 \mathrm{NH}_{2}\right.$, exchangable), 7.5-8.4 (m, $16 \mathrm{H}, \quad \mathrm{Ar}-\mathrm{H}) .{ }^{13} \mathrm{C}-\mathrm{NMR}$ (DMSO-d ${ }_{6}$ ): 75.6(2), 113.0(2), 116.7(4), 122.8(2), 124.8 (4), 125.1(2), 128.8(4), 129.8(2), 134.5(2), 136.0(2), 137.2(2), 138.4(2), 147.7(2), 156.9(2), 159.1(2), 165.5(2). Anal. Calcd. for $\mathrm{C}_{38} \mathrm{H}_{20} \mathrm{~N}_{10} \mathrm{O}_{8} \mathrm{~S}(776.69)$ : C, 58.76; $\mathrm{H}, 2.60 ; \mathrm{N}, 18.03$. Found: C, 58.90; H, 2.91; N, 17.89.

$1,1^{\prime}$-(4,4'-sulfonylbis $(4,1$-phenylene) $)$ bis (6-amino-4(4-nitrophenyl)-2-oxo-1,2-dihydropyridine-3,5-dicarbonitrile) 8 Yield $72 \%$, melting point $208.0^{\circ} \mathrm{C}$. IR: $v_{\max } / \mathrm{cm}^{-1}$ 3371, $3210\left(2 \mathrm{NH}_{2}\right), 3100(\mathrm{CH}$ arom.), 2194 $(2 \mathrm{C} \equiv \mathrm{N})$, $1674(2 \mathrm{C}=\mathrm{O}), 1346,1149\left(\mathrm{SO}_{2}\right), 1593,1332\left(\mathrm{NO}_{2}\right)$. ${ }^{1} \mathrm{H}-\mathrm{NMR} \quad\left(\mathrm{DMSO}-\mathrm{d}_{6}, \mathrm{D}_{2} \mathrm{O}\right): \delta \quad 6.7 \quad\left(\mathrm{~s}, \quad 4 \quad \mathrm{H}, \quad 2 \mathrm{NH}_{2}\right.$, exchangable), 7.8-8.4 (m, $16 \mathrm{H}, \quad$ Ar-H). ${ }^{13} \mathrm{C}-\mathrm{NMR}$ (DMSO-d $)$ : 73.7(2), 114.4(2), 114.9(4), 124.5(4), 127.9 (4), 129.1(4), 130.2(4), 137.6(2), 138.9(2), 141.1(2), 144.6 (2), 156.8(2), 157.3(2), 166.9(2). Anal. Calcd. for $\mathrm{C}_{38} \mathrm{H}_{20} \mathrm{~N}_{10} \mathrm{O}_{8} \mathrm{~S}(776.69): \mathrm{C}, 58.76 ; \mathrm{H}, 2.60 ; \mathrm{N}, 18.03$. Found: C, 59.00; H, 2.78; N, 18.09 .

1,1 '-(4,4'-sulfonylbis (4,1-phenylene))bis(6-amino-2oxo-4-(2,3,4-trimethoxyphenyl)-1,2-dihydropyridine-3,5dicarbonitrile) 9 Yield $70 \%$, melting point $286.4^{\circ} \mathrm{C}$. IR: $v_{\max } \cdot \mathrm{cm}^{-1} 3378,3210$ (2 $\left.\mathrm{NH}_{2}\right), 3097$ ( $\mathrm{CH}$ arom.), 2943, 2839(CH aliph.), 2218 (2 $\mathrm{C} \equiv \mathrm{N}), 1674(2 \mathrm{C}=\mathrm{O})$, 1390, $1157\left(\mathrm{SO}_{2}\right) .{ }^{1} \mathrm{H}-\mathrm{NMR}\left(\mathrm{DMSO}_{6} \mathrm{~d}_{6}, \mathrm{D}_{2} \mathrm{O}\right): \delta 3.7,3.8$ $\left(2 \mathrm{~s}, 18 \mathrm{H}, 6 \mathrm{OCH}_{3}\right), 6.7$ (s, $4 \mathrm{H}, 2 \mathrm{NH}_{2}$, exchangable), 6.9-8.0 (m, $12 \mathrm{H}, \mathrm{Ar}-\mathrm{H}) .{ }^{13} \mathrm{C}-\mathrm{NMR}\left(\mathrm{DMSO}_{\mathrm{d}}\right)$ : 55.8(2), 55.9(2), 60.5(2), 76.7(2), 107.7(2), 113.3(2), 115.4(2), 116.1(4), 121.1(2), 124.9(4), 129.8(4), 130.6(2), 138.9(2), 141.8(2), 150.1 (2), 153.5(2), 156.4(2), 159.6(2), 165.9(2). Anal. Calcd. for $\mathrm{C}_{44} \mathrm{H}_{34} \mathrm{~N}_{8} \mathrm{O}_{10} \mathrm{~S}(866.85)$ : C, 60.96; $\mathrm{H}$, 3.95; N, 12.93. Found: C, 60.72; H, 4.03; N, 12.81.

$1,1^{\prime}$-(4,4'-sulfonylbis (4,1-phenylene))bis(6-amino-4(benzo[d][1,3]dioxol-5-yl)-2-oxo-1,2-dihydropyridine3,5-dicarbonitrile) 10 Yield 77\%, melting point $291.6^{\circ} \mathrm{C}$. IR: $v_{\text {max }} / \mathrm{cm}^{-1}$ 3313, $3197\left(2 \mathrm{NH}_{2}\right), 3100$ (CH arom.), 2912, 2836( $\mathrm{CH}$ aliph.), 2214 (2 $\mathrm{C} \equiv \mathrm{N}), 1686(2 \mathrm{C}=\mathrm{O})$, 1390, $1195\left(\mathrm{SO}_{2}\right){ }^{1} \mathrm{H}-\mathrm{NMR} \quad\left(\mathrm{DMSO}_{6}, \mathrm{~d}_{2} \mathrm{O}\right): \delta \quad 6.1$ (s, $\left.4 \mathrm{H}, 2 \mathrm{CH}_{2}\right), 6.3\left(\mathrm{~s}, 4 \mathrm{H}, 2 \mathrm{NH}_{2}\right.$, exchangable), 7.0-8.0 (m, $14 \mathrm{H}, \mathrm{Ar}-\mathrm{H}) .{ }^{13} \mathrm{C}-\mathrm{NMR}\left(\mathrm{DMSO}-\mathrm{d}_{6}\right):$ 79.1(2), 102.4 (2), 109.1(2), 113.9(2), 115.7(2), 116.2(4), 120.5(2), 125.7 (4), 128.3(2), 128.4(4), 136.0(4), 148.1(4), 151.2(2), 161.3 (2), 164.9 (2). Anal. Calcd. for $\mathrm{C}_{40} \mathrm{H}_{22} \mathrm{~N}_{8} \mathrm{O}_{8} \mathrm{~S}(774.72)$ : C, 62.01; H, 2.86; N, 14.46. Found: C, 61.88; H, 2.94; N, 14.30 .

$1,1^{\prime}$-(4,4'-sulfonylbis(4,1-phenylene))bis(6-amino-4(2-chlorophenyl)-2-oxo-1,2-dihydropyridine-3,5-dicarbonitrile) 11 Yield $69 \%$, melting point $251.4^{\circ} \mathrm{C}$. IR: $v_{\max } /$ $\mathrm{cm}^{-1}$ 3380, $3213\left(2 \mathrm{NH}_{2}\right), 3097$ (CH arom.), $2222(2 \mathrm{C} \equiv \mathrm{N})$, $1678(2 \mathrm{C}=\mathrm{O}), 1380,1153\left(\mathrm{SO}_{2}\right), 740(\mathrm{C}-\mathrm{Cl}) .{ }^{1} \mathrm{H}-\mathrm{NMR}$
(DMSO- $\left.\mathrm{d}_{6}, \mathrm{D}_{2} \mathrm{O}\right): \delta 6.6$ (s, $4 \mathrm{H}, 2 \mathrm{NH}_{2}$, exchangable), 7.4-7.9 (m, $16 \mathrm{H}, \mathrm{Ar}-\mathrm{H}) .{ }^{13} \mathrm{C}-\mathrm{NMR}\left(\mathrm{DMSO}-\mathrm{d}_{6}\right)$ : 76.2(2), 114.8(2), 115.4(4), 124.6(4), 128.7(2), 129.8(2), 130.1(4), 130.3(2), 130.6(2), 131.7(2), 133.8(2), 138.7(2), 141.9(2), 153.7(2), 156.7(2), 159.4(2). Anal. Calcd. for $\mathrm{C}_{38} \mathrm{H}_{20} \mathrm{Cl}_{2} \mathrm{~N}_{8} \mathrm{O}_{4} \mathrm{~S}$ (755.59): C, 60.40; H, 2.67; N, 14.83. Found: C, 60.32; H, $2.79 ; \mathrm{N}, 14.61$.

$1,1^{\prime}$-(4,4'-sulfonylbis $(4,1$-phenylene) $)$ bis(6-amino-4(4-chlorophenyl)-2-oxo-1,2-dihydropyridine-3,5-

dicarbonitrile) 12 Yield $86 \%$, melting point $313.1^{\circ} \mathrm{C}$. IR: $v_{\max } \cdot / \mathrm{cm}^{-1}$ 3387, $3329\left(2 \mathrm{NH}_{2}\right), 3093$ (CH arom.), 2187 $(2 \mathrm{C} \equiv \mathrm{N}), 1660(2 \mathrm{C}=\mathrm{O}), 1370,1161\left(\mathrm{SO}_{2}\right), 771(\mathrm{C}-\mathrm{Cl})$. ${ }^{1} \mathrm{H}-\mathrm{NMR}\left(\mathrm{DMSO}-\mathrm{d}_{6}, \mathrm{D}_{2} \mathrm{O}\right): \delta 6.6\left(\mathrm{~s}, 4 \mathrm{H}, 2 \mathrm{NH}_{2}\right.$, exchangable), 7.5-8.1 (m, $16 \mathrm{H}, \mathrm{Ar}-\mathrm{H}) .{ }^{13} \mathrm{C}-\mathrm{NMR}\left(\mathrm{DMSO}-\mathrm{d}_{6}\right)$ : 80.2(2), 114.2(2), 115.6(4), 123.6(4), 128.7(4), 128.9(4), 130.0(4), 130.1(4), 134.8(4), 158.8(2), 153.9(2), 163.4(2). Anal. Calcd. for $\mathrm{C}_{38} \mathrm{H}_{20} \mathrm{Cl}_{2} \mathrm{~N}_{8} \mathrm{O}_{4} \mathrm{~S}$ (755.59): C, 60.40; H, 2.67; N, 14.83. Found: C, 60.71; H, 2.38; N, 15.08.

1,1 '-(4,4'-sulfonylbis (4,1-phenylene))bis(6-amino-4(4-(dimethylamino)phenyl)-2-oxo-1,2-dihydropyridine-3,5-dicarbonitrile) 13 Yield 71\%, melting point $277.4^{\circ} \mathrm{C}$. IR: $\mathrm{v}_{\max } / \mathrm{cm}^{-1} 3464,3367\left(2 \mathrm{NH}_{2}\right), 3097(\mathrm{CH}$ arom.), 2908, 2870 ( $\mathrm{CH}$ aliph.), $2210(2 \mathrm{C} \equiv \mathrm{N}), 1678$ $(2 \mathrm{C}=\mathrm{O}), 1381,1168\left(\mathrm{SO}_{2}\right){ }^{1} \mathrm{H}-\mathrm{NMR}\left(\mathrm{DMSO}-\mathrm{d}_{6}, \mathrm{D}_{2} \mathrm{O}\right): \delta$ 3.0 (s, $\left.12 \mathrm{H}, 4 \mathrm{CH}_{3}\right), 6.8$ (s, $4 \mathrm{H}, 2 \mathrm{NH}_{2}$, exchangable), 7.5-8.0 (m, $16 \mathrm{H}, \mathrm{Ar}-\mathrm{H}) .{ }^{13} \mathrm{C}-\mathrm{NMR}\left(\mathrm{DMSO}-\mathrm{d}_{6}\right)$ : 40.1(4), 78.1(2), 112.9(4), 117.8(2), 118.4(4), 120.2(2), 125.8(4), 128.2(4), 129.2 (4), 127.6(2), 143.2(2), 151.4(2), 153.4(2), 162.2(2), 162.3(2). Anal. Calcd. for $\mathrm{C}_{42} \mathrm{H}_{32} \mathrm{~N}_{10} \mathrm{O}_{4} \mathrm{~S}(772.83)$ : C, 65.27; H, 4.17; N, 18.12. Found: C, 65.50; H, 4.00; N, 18.41 .

1,1 '-(4,4'-sulfonylbis $(4,1$-phenylene) $)$ bis(6-amino-4(2-methoxyphenyl)-2-oxo-1,2-dihydropyridine-3,5dicarbonitrile) 14 Yield $70 \%$, melting point $269.6^{\circ} \mathrm{C}$. IR: $v_{\text {max }} \cdot / \mathrm{cm}^{-1}$ 3448, 3367 (2 $\left.\mathrm{NH}_{2}\right), 3066$ ( $\mathrm{CH}$ arom.), 2935, 2870 ( $\mathrm{CH}$ aliph.), $2183(2 \mathrm{C} \equiv \mathrm{N}), 1678$ (2 C=O), 1350, $1149\left(\mathrm{SO}_{2}\right) .{ }^{1} \mathrm{H}-\mathrm{NMR}\left(\mathrm{DMSO}-\mathrm{d}_{6}, \mathrm{D}_{2} \mathrm{O}\right): \delta 3.9(\mathrm{~s}$, $\left.6 \mathrm{H}, 2 \mathrm{OCH}_{3}\right), 6.6\left(\mathrm{~s}, 4 \mathrm{H}, 2 \mathrm{NH}_{2}\right.$, exchangable), 7.3-8.1 (m, $16 \mathrm{H}, \mathrm{Ar}-\mathrm{H})$. Anal. Calcd. for $\mathrm{C}_{40} \mathrm{H}_{26} \mathrm{~N}_{8} \mathrm{O}_{6} \mathrm{~S}(746.75)$ : C, 64.34; H, 3.51; N, 15.01. Found: C, 64.77; H, 3.31; N, 15.36 .

$1,1^{\prime}$-(4,4'-sulfonylbis (4,1-phenylene))bis(6-amino-4(4-methoxyphenyl)-2-oxo-1,2-dihydropyridine-3,5dicarbonitrile) 15 Yield $76 \%$, melting point $303.8^{\circ} \mathrm{C}$. IR: $v_{\max } \cdot / \mathrm{cm}^{-1} 3317,3197$ (2 $\left.\mathrm{NH}_{2}\right), 3070$ ( $\mathrm{CH}$ arom.), 2339, 2843 ( $\mathrm{CH}$ aliph.), 2214 (2 $\mathrm{C} \equiv \mathrm{N}), 1686(2 \mathrm{C}=\mathrm{O})$, 1370, $1149\left(\mathrm{SO}_{2}\right) .{ }^{1} \mathrm{H}-\mathrm{NMR}$ (DMSO-d $\left.\mathrm{D}_{6} \mathrm{D}_{2} \mathrm{O}\right): \delta 3.9(\mathrm{~s}$, $\left.6 \mathrm{H}, 2 \mathrm{OCH}_{3}\right), 6.3\left(\mathrm{~s}, 4 \mathrm{H}, 2 \mathrm{NH}_{2}\right.$, exchangable), 7.0-8.3 (m, $16 \mathrm{H}, \mathrm{Ar}-\mathrm{H}) .{ }^{13} \mathrm{C}-\mathrm{NMR}\left(\mathrm{DMSO}-\mathrm{d}_{6}\right)$ : 56.0(2), 80.2(2), 
105.4(2), 114.2(2), 115.6(4), 123.4(4), 123.8(4), 128.6(4), 131.3(4), 133.1(4), 133.6(2), 134.2(4), 134.8(2), 151.3(2), 152.8(2), 153.8(2), 163.4(2). Anal. Calcd. for $\mathrm{C}_{40} \mathrm{H}_{26} \mathrm{~N}_{8} \mathrm{O}_{6} \mathrm{~S}$ (746.75): C, 64.34; H, 3.51; N, 15.01. Found: C, 64.48; $\mathrm{H}, 3.70 ; \mathrm{N}, 14.92$.

$1,1^{\prime}$-(4,4'-sulfonylbis $(4,1$-phenylene) $)$ bis (6-amino-2oxo-4-(thiophen-2-yl)-1,2-dihydropyridine-3,5-dicarbonitrile) 16 Yield $81 \%$, melting point $187.7^{\circ} \mathrm{C}$. IR: $v_{\max } \cdot / \mathrm{cm}^{-}$ 1 3375, $3213\left(2 \mathrm{NH}_{2}\right), 3100$ ( $\mathrm{CH}$ arom.), $2214(2 \mathrm{C} \equiv \mathrm{N})$, $1676(2 \mathrm{C}=\mathrm{O}), 1390,1149\left(\mathrm{SO}_{2}\right) .{ }^{1} \mathrm{H}-\mathrm{NMR}\left(\mathrm{DMSO}_{\mathrm{d}}\right.$, $\left.\mathrm{D}_{2} \mathrm{O}\right): \delta 6.6\left(\mathrm{~s}, 4 \mathrm{H}, 2 \mathrm{NH}_{2}\right.$, exchangable), 7.2-8.7 (m, $14 \mathrm{H}, \mathrm{Ar}-\mathrm{H}) .{ }^{13} \mathrm{C}-\mathrm{NMR}\left(\mathrm{DMSO}-\mathrm{d}_{6}\right):$ 75.3(2), 116.2(4), 120.5(2), 127.2(2), 127.7(2), 128.7(2), 129.8(4), 130.4(2), 135.6(2), 138.1(2), 144.6(2), 153.5(2), 162.1(2), 171.9(2). Anal. Calcd. for $\mathrm{C}_{34} \mathrm{H}_{18} \mathrm{~N}_{8} \mathrm{O}_{4} \mathrm{~S}_{3}(698.75)$ : C, 58.44; $\mathrm{H}$, 2.60; N, 16.04. Found: C, 58.19; H, 2.90; N, 16.32 .

$1,1^{\prime}$-(4,4'-sulfonylbis (4,1-phenylene))bis(6-amino-2oxo-1,2-dihydropyridine-3,5-dicarbonitrile) 17 Yield $66 \%$, melting point $308.9^{\circ} \mathrm{C}$. IR: $v_{\max } / \mathrm{cm}^{-1} 3371,3206(2$ $\left.\mathrm{NH}_{2}\right), 2187(2 \mathrm{C} \equiv \mathrm{N}), 1680 \quad(2 \mathrm{C}=\mathrm{O}), 1377,1145$ $\left(\mathrm{SO}_{2}\right) .{ }^{1} \mathrm{H}-\mathrm{NMR}\left(\mathrm{DMSO}_{6}, \mathrm{~d}_{6} \mathrm{O}\right): \delta 6.8\left(\mathrm{~s}, 4 \mathrm{H}, 2 \mathrm{NH}_{2}\right.$, exchangable), 7.6-7.8 ( $\mathrm{m}, 10 \mathrm{H}, \mathrm{Ar}-\mathrm{H}+2 \mathrm{CH}$ pyridone). ${ }^{13}$ C-NMR(DMSO-d $\left.{ }_{6}\right): 62.9(2), 100.2(2), 116.2(4), 122.9$ (4), 130.2(4), 136.9(2), 139.6(2), 151.7(2), 154.2(2), 158.7 (2). Anal. Calcd. for $\mathrm{C}_{26} \mathrm{H}_{14} \mathrm{~N}_{8} \mathrm{O}_{4} \mathrm{~S}(534.51)$ : C, 58.42; $\mathrm{H}$, 2.64; N, 20.96. Found: C, 58.60; H, 2.88; N, 20.71.

$1,1^{\prime}$-(4,4'-sulfonylbis $(4,1$-phenylene $\left.)\right)$ bis(6-amino-4methyl-2-oxo-1,2-dihydropyridine-3,5-dicarbonitrile) 18 Yield $68 \%$, melting point $230.2^{\circ} \mathrm{C}$. IR: $\mathrm{v}_{\max } \cdot / \mathrm{cm}^{-1} 3410$, $3394\left(2 \mathrm{NH}_{2}\right), 2935,2860$ ( $\mathrm{CH}$ aliph.), $2198(2 \mathrm{C} \equiv \mathrm{N})$, $1686(2 \mathrm{C}=\mathrm{O}), 1390,1149\left(\mathrm{SO}_{2}\right) .{ }^{1} \mathrm{H}-\mathrm{NMR}\left(\mathrm{DMSO}-\mathrm{d}_{6}\right.$, $\left.\mathrm{D}_{2} \mathrm{O}\right): \delta 1.6\left(\mathrm{~s}, 6 \mathrm{H}, 2 \mathrm{CH}_{3}\right), 6.7\left(\mathrm{~s}, 4 \mathrm{H}, 2 \mathrm{NH}_{2}\right.$, exchangable), 7.3-8.0 (m, $8 \mathrm{H}, \mathrm{Ar}-\mathrm{H}) .{ }^{13} \mathrm{C}-\mathrm{NMR}\left(\mathrm{DMSO}-\mathrm{d}_{6}\right): 8.4$ (2), 56.2(2), 114.9(2), 116.2(4), 119.5(4), 128.1(4), 136.7 (2), 136.9(2), 151.2(2), 152.8(2), 166.9(2). Anal. Calcd. for $\mathrm{C}_{28} \mathrm{H}_{18} \mathrm{~N}_{8} \mathrm{O}_{4} \mathrm{~S}(562.56)$ : C, 59.78; H, 3.23; N, 19.92. Found: C, 59.54; H, 3.40; N, 19.69.

1,1'-(4,4'-sulfonylbis(4,1-phenylene))bis(6-amino-4butyl-2-oxo-1,2-dihydropyridine-3,5-dicarbonitrile)19 Yield $62 \%$, melting point $236.3^{\circ} \mathrm{C}$. IR: $v_{\text {max }} / \mathrm{cm}^{-1} 3380$, $3367\left(2 \mathrm{NH}_{2}\right), 2954,2840$ ( $\mathrm{CH}$ aliph.), $2195(2 \mathrm{C} \equiv \mathrm{N})$, $1650(2 \mathrm{C}=\mathrm{O}), 1399,1149\left(\mathrm{SO}_{2}\right) .{ }^{1} \mathrm{H}-\mathrm{NMR}\left(\mathrm{DMSO}_{6} \mathrm{~d}_{6}\right.$, $\left.\mathrm{D}_{2} \mathrm{O}\right): \delta 1.1\left(\mathrm{t}, 6 \mathrm{H}, 2 \mathrm{CH}_{3}\right), 1.3-2\left(\mathrm{~m}, 12 \mathrm{H}, 6 \mathrm{CH}_{2}\right), 6.6(\mathrm{~s}$, $4 \mathrm{H}, 2 \mathrm{NH}_{2}$, exchangable), 7.3-7.9 (m, $\left.8 \mathrm{H}, \mathrm{Ar}-\mathrm{H}\right) .{ }^{13} \mathrm{C}-$ NMR(DMSO-d 6 ): 13.5(2), 21.7(2), 22.3(2), 29.1(2), 62.6 (2), 113.5(2), 113.9(4), 120.8(4), 129.1(4), 133.9(2), 142.3(2), 153.6(2), 161.6(2), 175.8(2). Anal. Calcd. for $\mathrm{C}_{34} \mathrm{H}_{30} \mathrm{~N}_{8} \mathrm{O}_{4} \mathrm{~S}$ (646.72): C, 63.14; H, 4.68; N, 17.33. Found: C, 63.00; H, 4.90; N, 17.01.
(2E,2'E)-N,N'-(4,4'-sulfonylbis(4,1-phenylene))bis(2cyano-3-(2,4-dichlorophenyl)acrylamide) 20 A mixture of 2 (3.82 g, $0.01 \mathrm{~mol}$.) and 2,4-dichlorobenzaldehyde ( $3.50 \mathrm{~g}, 0.02 \mathrm{~mol}$.) in acetic acid was refluxed for $8 \mathrm{~h}$, the obtained solid was filtered and recrystallized from acetic acid to give 20 . Yield $88 \%$, melting point $253.4^{\circ} \mathrm{C}$. IR: $v_{\max } / \mathrm{cm}^{-1} 3372(2 \mathrm{NH}), 3986$ (CH arom.), 2940, 2860 ( $\mathrm{CH}$ aliph.), $2203(2 \mathrm{C} \equiv \mathrm{N}), 1652(2 \mathrm{C}=\mathrm{O}), 1390,1145$ $\left(\mathrm{SO}_{2}\right), 829(\mathrm{C}-\mathrm{Cl}) .{ }^{1} \mathrm{H}-\mathrm{NMR}$ (DMSO-d, $\left.\mathrm{D}_{2} \mathrm{O}\right): \delta 6.9-7.8$ (m, 14 H, Ar- $\mathrm{H}), 10.0$ (s, $2 \mathrm{H}, 2 \mathrm{NH}$, exchangable). ${ }^{13} \mathrm{C}$ NMR(DMSO-d ${ }_{6}$ ): 112.8(2), 119.0(2), 122.8(4), 127.9(2), 128.5 (4), 129.0(2), 130.1(2), 130.4(2), 132.6(2), 134.6(2), 135.9(2), 142.8(2), 153.3(2), 163.3(2). Anal. Calcd. for $\mathrm{C}_{32} \mathrm{H}_{18} \mathrm{C}_{44} \mathrm{~N}_{4} \mathrm{O}_{4} \mathrm{~S}$ (696.39): C, 55.19; H, 2.61; N, 8.05. Found: C, 55.36; H, 2.50; N, 7.99 .

N,N'-(4,4'-sulfonylbis(4,1-phenylene))bis(2-oxo-2 Hchromene-3-carboxamide) 21 To a solution of 2 (3.82 g, $0.01 \mathrm{~mol}$.) in acetic anhydride $(30 \mathrm{~mL})$, salicylaldehyde ( $2.44 \mathrm{~g}, 0.02 \mathrm{~mol}$.) and fused $\mathrm{Na}$ acetate $(1.6 \mathrm{~g}, 0.02 \mathrm{~mol}$.) were added, the reaction mixture was refluxed for $3 \mathrm{~h}$, cooled and the solid obtained was crystallized from dioxane to give 21 . Yield $59 \%$, melting point $195.5^{\circ} \mathrm{C}$. IR: $v_{\max } \cdot \mathrm{cm}^{-1} 3433$ (2 NH), 3097 (CH arom.), 1762, $1720(4 \mathrm{C}=\mathrm{O}), 1396,1157\left(\mathrm{SO}_{2}\right) .{ }^{1} \mathrm{H}-\mathrm{NMR}$ (DMSO-d 6 , $\left.\mathrm{D}_{2} \mathrm{O}\right): \delta$ 7.3-7.6 (m, $\left.16 \mathrm{H}, \mathrm{Ar}-\mathrm{H}\right), 8.2$ (s, $\left.2 \mathrm{H}, 2 \mathrm{CH}\right), 10.4$ (s, $2 \mathrm{H}, 2 \mathrm{NH}$, exchangable). ${ }^{13} \mathrm{C}-\mathrm{NMR}\left(\mathrm{DMSO}-\mathrm{d}_{6}\right): 118.9(2)$, 121.8(2), 123.3(2), 124.4(4), 126.4(2), 127.7(2), 128.8(2), 130.7(4), 137.6(2), 141.2(2), 144.3(2), 152.1(2), 169.1(2), 171.9(2). Anal. Calcd. for $\mathrm{C}_{32} \mathrm{H}_{20} \mathrm{~N}_{2} \mathrm{O}_{8} \mathrm{~S}(592.57)$ :C, 64.86; $\mathrm{H}$, 3.40; N, 4.73. Found: C, 64.76; H, 3.31; N, 5.00.

N,N'-(4,4'-sulfonylbis(4,1-phenylene))bis(2-imino2 H-chromene-3-carboxamide) 22 A mixture of compound 2 (3.82 g, $0.01 \mathrm{~mol}$.), salicylaldehyde (2.44 g, $0.02 \mathrm{~mol}$.) and anhydrous ammounium acetate $(2.30 \mathrm{~g}$, $0.03 \mathrm{~mol}$.) was refluxed in ethanol $(50 \mathrm{~mL})$ for $2 \mathrm{~h}$. The solid obtained was crystallized from ethanol to give 22 . Yield $64 \%$, melting point $256.1^{\circ} \mathrm{C}$. IR: $v_{\max } \cdot / \mathrm{cm}^{-1} 3383$, 3259 (4 NH), 1686 (2 C=O), 1377, $1149\left(\mathrm{SO}_{2}\right) .{ }^{1} \mathrm{H}-\mathrm{NMR}$ $\left(\right.$ DMSO- $\left._{6}, \mathrm{D}_{2} \mathrm{O}\right): \delta$ 7.2-8.0 (m, $\left.16 \mathrm{H}, \mathrm{Ar}-\mathrm{H}\right), 8.6(\mathrm{~s}, 2 \mathrm{H}$, $2 \mathrm{CH}$ ), 10.4 (s, $2 \mathrm{H}, 2 \mathrm{NH}$, exchangable), 13.1 (s, $2 \mathrm{H}, 2 \mathrm{NH}$, exchangable), ${ }^{13}$ C-NMR(DMSO-d 6 ): 114.9(2), 119.7(2), 122.2 (2), 122.5(4), 125.7(2), 127.9(2), 128.8(2), 129.4(4), 134.1(2), 141.6(2), 142.1(2), 153.7(2), 160.2(2), 165.5(2). Anal. Calcd. for $\mathrm{C}_{32} \mathrm{H}_{22} \mathrm{~N}_{4} \mathrm{O}_{6} \mathrm{~S}(590.61): \mathrm{C}, 65.08 ; \mathrm{H}, 3.75 ; \mathrm{N}, 9.49$. Found: C, 65.23; H, 3.60; N, 9.29.

\section{General procedure for synthesis of compound 23 and 24}

Equimolar amount of compound 21or 22 (0.01 mol.) and malononitrile (1.32 g, $0.02 \mathrm{~mol}$.) and anhydrous ammonium acetate $(2.30 \mathrm{~g}, 0.03 \mathrm{~mol}$.) were refluxed in ethanol $(50 \mathrm{~mL})$ for $5 \mathrm{~h}$. The obtained solid was crystallized from dioxane to give 23 and 24 , respectively. 
3,3'-(4,4'-sulfonylbis(4,1-phenylene))bis(2-amino-4,5dioxo-4,5-dihydro-3 H-chromeno[3,4-c]pyridine-1carbonitrile) 23 Yield $75 \%$, melting point $267.8^{\circ} \mathrm{C}$. IR: $v_{\text {max }} / / \mathrm{cm}^{-1} 3444,3344\left(2 \mathrm{NH}_{2}\right), 3100$ ( $\mathrm{CH}$ arom.), $2199(2 \mathrm{C} \equiv \mathrm{N}), 1690,1660(4 \mathrm{C}=\mathrm{O}), 1373,1153\left(\mathrm{SO}_{2}\right)$. ${ }^{1} \mathrm{H}-\mathrm{NMR}\left(\mathrm{DMSO}-\mathrm{d}_{6}, \mathrm{D}_{2} \mathrm{O}\right): \delta 6.7\left(\mathrm{~s}, 4 \mathrm{H}, 2 \mathrm{NH}_{2}\right.$, exchangable), 7.1-8.0 (m, $16 \mathrm{H}, \mathrm{Ar}-\mathrm{H}) .{ }^{13} \mathrm{C}-\mathrm{NMR}\left(\mathrm{DMSO}-\mathrm{d}_{6}\right): 76.7$ (2), 115.8(2), 119.0(2), 121.8(2), 122.6(4), 123.9(2), 124.8 (2), 128.3(4), 129.0(2), 135.0(4), 141.1(2), 143.5(2), 158.0 (2), 158.4(2), 164.8(2), 169.1(2), 170.3(2). Anal. Calcd. for $\mathrm{C}_{38} \mathrm{H}_{20} \mathrm{~N}_{6} \mathrm{O}_{8} \mathrm{~S}(720.67)$ : C, 63.33; H, 2.80; N, 11.66. Found: C, 63.11; H, 2.96; N, 11.49.

3,3'-(4,4'-sulfonylbis (4,1-phenylene))bis(2-amino-5imino-4-oxo-4,5-dihydro-3 H-chromeno[3,4-c]pyridine1-carbonitrile) 24 Yield $77 \%$, melting point $>360^{\circ} \mathrm{C}$. IR: $v_{\max } \cdot / \mathrm{cm}^{-1} 3441,3348,3236,3186\left(2 \mathrm{NH}, 2 \mathrm{NH}_{2}\right), 2203$ $(2 \mathrm{C} \equiv \mathrm{N}), 1680(2 \mathrm{C}=\mathrm{O}), 1381,1153\left(\mathrm{SO}_{2}\right) .{ }^{1} \mathrm{H}-\mathrm{NMR}$ (DMSO- $\left.\mathrm{d}_{6}, \mathrm{D}_{2} \mathrm{O}\right): \delta 6.6\left(\mathrm{~s}, 4 \mathrm{H}, 2 \mathrm{NH}_{2}\right.$, exchangable), 7.1-7.9 (m, $16 \mathrm{H}, \mathrm{Ar}-\mathrm{H}), 8.9\left(\mathrm{~s}, 2 \mathrm{H}, 2 \mathrm{NH}\right.$, exchangable). ${ }^{13} \mathrm{C}-\mathrm{NMR}$ (DMSO- $\mathrm{d}_{6}$ ): 78.1(2), 112.6(2), 116.7(4), 122.4(2), 122.8(2), 124.6(4), 128.1(2), 128.9(4), 129.1(2), 137.4(2), 155.6(2), 156.1 (2), 158.7(2), 159.6(2), 163.5(2). Anal. Calcd. for $\mathrm{C}_{38} \mathrm{H}_{22} \mathrm{~N}_{8} \mathrm{O}_{6} \mathrm{~S}$ (718.70):C, 63.50; H, 3.09; N, 15.59. Found: C, 63.44; H, 3.18; N, 15.75 .

\section{Molecular docking}

All the molecular modeling studies were carried out on an Intel Pentium 1.6 GHz processor, $512 \mathrm{MB}$ memory with Windows XP operating system using Molecular Operating Environment (MOE, 10.2008) software. All the minimizations were performed with MOE until a RMSD gradient of $0.05 \mathrm{kcal} \mathrm{mol}^{-1} \mathrm{~A}^{\mathrm{o}-1}$ with MMFF94X force field and the partial charges were automatically calculated. The X-ray crystallographic structure of franesyltransferase and arginine methyltransferase (PRMT1) complexes with their ligands (PDB ID: 3E30, 3Q7E) were obtained from the protein data bank. The enzymes were prepared for docking studies where: (i) Ligand molecule was removed from the enzyme active site. (ii) Hydrogen atoms were added to the structure with their standard geometry. (iii) MOE Alpha Site Finder was used for the active sites search in the enzyme structure and dummy atoms were created from the obtained alpha spheres. (iv) The obtained model was then used in predicting the ligand enzymes interactions at the active site.

\section{In vitro antitumor activity}

Human tumor breast cell line (MCF7) was used in this study. The cytotoxic activity was measured in vitro for the newly synthesized compounds using the SulfoRhodamine-B stain (SRB) assay using the method of Skehan et al. [52]. The in vitro anticancer screening was done by the pharmacology unit at the National Cancer Institute, Cairo University.

Cells were plated in 96-multiwell plate (104 cells/well) for $24 \mathrm{~h}$ before treatment with the compound(s) to allow attachment of cell to the wall of the plate. Test compounds were dissolved in dimethyl sulfoxide. Different concentrations of the compound under test $(10,25,50$, and $100 \mu \mathrm{M})$ were added to the cell monolayer. Triplicate wells were prepared for each individual concentration. Monolayer cells were incubated with the compound(s) for $48 \mathrm{~h}$ at $37^{\circ} \mathrm{C}$ and in atmosphere of $5 \% \mathrm{CO}_{2}$. After $48 \mathrm{~h}$, cells were fixed, washed and stained for $30 \mathrm{~min}$ with $0.4 \%$ (wt/vol) SRB dissolved in 1\% acetic acid. Excess unbound dye was removed by four washes with $1 \%$ acetic acid and attached stain was recovered with Trise-EDTA buffer. Color intensity was measured in an ELISA reader. The relation between surviving fraction and drug concentration is plotted to get the survival curve for breast tumor cell line after the specified time. The molar concentration required for $50 \%$ inhibition of cell viability $\left(\mathrm{IC}_{50}\right)$ was calculated and compared to the reference drug Doxorubicin (CAS, 25316-40-9). The surviving fractions were expressed as means \pm standard error and the results are given in Table 3.

\section{Conclusions}

Diarylsulfone derivatives may serve as good candidates in the search for novel anticancer agents as illustrated by the $\mathrm{IC}_{50}$ values of the investigated compounds. These values were better than that of Doxorubicin. The mechanism of action as anticancer of the synthesized compounds was investigated through molecular docking on the active site of farnesyl transferase and arginine methyltransferase. Both enzymes could be the target of action of these compounds based on the good energy scores and amino acid interactions in the active sites of enzymes however, the exact mechanism of action still needs more investigation to be clarified.

\section{Competing interests}

The authors declare that they have no competing interests.

\section{Authors' contributions}

M.Al-Said, M.Ghorab designed the synthetic schemes for all synthesized compounds. All authors contributed in the chemical synthesis. Y.Nissan carried out molecular docking and interpretation of its results as well as interpretation of the biological results. All authors read and approved the final manuscript.

\section{Acknowledgement}

The authors are grateful to the sponsorship of the College of Pharmacy Research Centre and the Deanship of the Scientific Research, King Saud University, Riyadh, Saudi Arabia.

\section{Author details}

${ }^{1}$ Medicinal, Aromatic and Poisonous Plants Research Center (MAPPRC), College of Pharmacy, King Saud University, 2457, Riyadh 11451, Saudi Arabia. ${ }^{2}$ Pharmaceutical Chemistry Department, Faculty of Pharmacy, Cairo University, Cairo, Egypt. 
Received: 14 April 2012 Accepted: 7 June 2012

Published: 2 July 2012

\section{References}

1. Cocco MT, Congiu C, Onnis V: Synthesis and antitumour activity of 4-hydroxy-2-pyridone derivatives. Euro J of Med Chem 2000, 35:545-552.

2. Fan N, Evans DB, Rank KB, Thomas RC, Tarpley WG, Sharma SK: Mechanism of resistance to U-90152 S and sensitization to L-697,661 by a proline to leucine change at residue 236 of human immunodeficiency virus type 1 (HIV-1) reverse transcriptase. FEBS Lett 1995, 359:233-238.

3. Emini EA, Staszewski S, Schneider CL, Waterbury JA, Schleif WA, Goehler R, Deussen A, Duerr S, Massari FE, Calandra GB, Hoffstedt B, Byrnes WW: 94 Combination therapy with AZT prevents selection of HIV-1 variants that are highly resistant to the nonnucleoside reverse transcriptase inhibitor L-697, 661. Antiviral Res 1993, 20:94.

4. Van Der Zypp A, Rechtman M, Majewski H: The role of cyclic nucleotides and calcium in the relaxation produced by amrinone in rat aorta. Gen Pharmacol 2000, 34:245-253.

5. Rechtman MP, Van Der Zypp A, Majewski H: Amrinone reduces ischaemiareperfusion injury in rat heart. Eur J Pharmacol 2000, 402:255-262.

6. Jeremy JY, Gill J, Mikhailidis D: Effect of milrinone on thromboxane A2 synthesis, cAMPphosphodiesterase and $45 \mathrm{Ca} 2+$ uptake by human platelets. Eur J Pharmacol 1993, 245:67-73.

7. Raffaeli S, Ferroni C, Spurgeon HA, Capogrossi MC: Milrinone enhances cytosolic calcium transient and contraction in rat cardiac myocytes during beta-adrenergic stimulation. Int I Cardiol 1989, 25:S63-S69.

8. Card JW, Racz WJ, Brien JF, Margolin SB, Massey TE: Differential effects of pirfenidone on acute pulmonary injury and ensuing fibrosis in the hamster model of amiodarone-induced pulmonary toxicity. Toxicological sciences an official journal of the Society of Toxicology 2003, 75:169-180.

9. Kehrer JP, Margolin SB: Pirfenidone diminishes cyclophosphamideinduced lung fibrosis in mice. Toxicol Lett 1997, 90:125-132.

10. Card JW, Lalonde BR, Rafeiro E, Tam AS, Racz WJ, Brien JF, Bray TM, Massey TE: Amiodarone-induced disruption of hamster lung and liver mitochondrial function: lack of association with thiobarbituric acidreactive substance production. Toxicol Lett 1998, 98:41-50.

11. Giri SN, Schwartz LW, Hollinger MA, Freywald ME, Schiedt MJ, Zuckerman JE: Biochemical and structural alterations of hamster lungs in response to intratracheal administration of bleomycin. Exp Mol Pathol 1980, 33:1-14.

12. Iyer SN, Wild JS, Schiedt MJ, Hyde DM, Margolin SB, Giri SN: Dietary intake of pirfenidone ameliorates bleomycin-induced lung fibrosis in hamsters. J Lab Clin Med 1995, 125:779-785.

13. Iyer SN, Gurujeyalakshmi G, Giri SN: Effects of pirfenidone on transforming growth factor-beta gene expression at the transcriptional level in bleomycin hamster model of lung fibrosis. J Pharmacol Exp Ther 1999, 291:367-373.

14. Iyer SN, Hyde DM, Giri SN: Anti-inflammatory effect of pirfenidone in the bleomycin-hamster model of lung inflammation. Inflamm 2000, 24:477-491.

15. Selman $M$, King $T E$, Pardo A: Idiopathic pulmonary fibrosis: prevailing and evolving hypotheses about its pathogenesis and implications for therapy. Ann Intern Med 2001, 134:136-151.

16. Kakugawa T, Mukae H, Hayashi T, Ishii H, Abe K, Fujii T, Oku H, Miyazaki M, Kadota J, Kohno S: Pirfenidone attenuates expression of HSP47 in murine bleomycin-induced pulmonary fibrosis. Eur Respir J 2004, 24:57-65.

17. Pemberton N, Pinkner JS, Jones JM, Jakobsson L, Hultgren SJ, Almqvist F: Bicyclic 2-pyridone targeting pilus biogenesis in uropathogenic $\mathrm{E}$ coli. Tetrahedron Lett 2007, 48:4543-4546.

18. Hamdy NA, Gamal-Eldeen AM: New pyridone, thioxopyridine, pyrazolopyridine and pyridine derivatives that modulate inflammatory mediators in stimulated RAW 264.7 murine macrophage. Eur J Med Chem 2009, 44:4547-4556.

19. Li Q, Wang W, Berst KB, Claiborne A, Hasvold L, Raye K, Tufano M, Nilius A, Shen LL, Flamm R, Alder J, Marsh K, Crowell D, Chu DT, Plattner J: Synthesis and structure-activity relationships of 2-pyridones: II. 8-(Fluoro-substituted pyrrolidinyl)-2-pyridones as antibacterial agents. Bioorg Med Chem Lett 1998, 8:1953-1958.
20. Vegi SR, Boovanahalli SK, Patro B, Mukkanti K: SPF32629A and SPF32629B: enantioselective synthesis, determination of absolute configuration, cytotoxicity and antibacterial evaluation. Eur J Med Chem 2011, 44:1803-1812

21. Dominguez JN, Leon C, Rodrigues J, Gamboa De Dominguez N, Gut J, Rosenthal PJ: Synthesis of chlorovinylsulfones as structural analogs of chalcones and their antiplasmodial activities. Eur J Med Chem 2009, 44:1457-1462.

22. Szzlágyi G, Somorai T, Bozó É, Langó J, Nagy G, Reiter J, Janáky J: Preparation and antiarthritic activity of new 1,5-diaryl-3-alkylthio-1 H1,2,4-triazoles and corresponding sulfoxides and sulfones. $\mathrm{H}-1,2,4$-triazoles and corresponding sulfoxides and sulfones. Eur J Med Chem 1990, 25:95-101.

23. Santelli-Rouvier C, Barret JM, Farrell CM, Sharples D, Hill BT, Barbe J: Synthesis of 9-acridinyl sulfur derivatives: sulfides, sulfoxides and sulfones. Comparison of their activity on tumour cells. Eur J Med Chem 2004, 39:1029-1038.

24. Murafuji T, Fujiwara Y, Yoshimatsu D, Miyakawa I, Migita K, Mikata Y: Bismuth heterocycles based on a diphenylsulfone scaffold: synthesis and substituent effect on the antifungal activity against Saccharomyces cerevisiae. Eur J Med Chem 2011, 46:519-525.

25. Usera AR, Dolan P, Kensler TW, Posner GH: Novel alkyl side chain sulfone 1alpha,25-dihydroxyvitamin D3 analogs: a comparison of in vitro antiproliferative activities and in vivo calcemic activities. Bioorg Med Chem 2009, 17:5627-5631.

26. Badal S, Williams SA, Huang G, Francis S, Vendantam P, Dunbar O, Jacobs $H$, Tzeng TJ, Gangem J, Delgoda R: Cytochrome P450 1 enzyme inhibition and anticancer potential of chromene amides from Amyrisplumieri. Fitoterapia 2011, 82:230-236.

27. Endo S, Matsunaga T, Kuwata K, Zhao HT, El-Kabbani O, Kitade Y, Hara A: Chromene-3-carboxamide derivatives discovered from virtual screening as potent inhibitors of the tumour maker, AKR1B10. Bioorg Med Chem 2010, 18:2485-2490.

28. Heo SJ, Kim KN, Yoon WJ, Oh C, Choi YU, Affan A, Lee YJ, Lee HS, Kang DH: Chromene induces apoptosis via caspase-3 activation in human leukemia HL-60 cells. Food Chem Toxico 2011, 49:1998-2004.

29. Rao RN, Suman P, Yogeeswari P, Sriram D, Basha T, Vardhan S: Synthesis, structure-activity relationship of novel substituted $4 \mathrm{H}$-chromen-1,2,3,4tetrahydropyrimidine-5-carboxylates as potential anti-mycobacterial and anticancer agents. Bioorg \& Med Chem Lett 2011, 21:2855-2859.

30. Santelli-Rouvier C, Barret JM, Farrell CM, Sharples D, Hill BT, Barbe J: Synthesis of 9-acridinyl sulfur derivatives: sulfides, sulfoxides and sulfones. Comparison of their activity on tumour cells. Eur J Med Chem 2004, 39:1029-1038.

31. El-Sayed AT: Synthesis of some novel pyrazolo[3,4-b]pyridine and pyrazolo[3,4-d]pyrimidine derivatives bearing 5,6-diphenyl-1,2,4-triazine moiety as potential antimicrobial agents. Eur I Med Chem 2009, 44:4385-92.

32. Onnis V, Cocco MT, Fadda R, Congiu C: Synthesis and evaluation of anticancer activity of 2-arylamino-6-trifluoromethyl-3(hydrazonocarbonyl)pyridines. Bioorg Med Chem 2009, 17:6158-65.

33. Karki R, Thapa P, Kang MJ, Jeong TC, Nam JM, Kim HL, Na Y, Cho WJ, Kwon Y, Lee ES: Synthesis, topoisomerase I and II inhibitory activity, cytotoxicity, and structure-activity relationship study of hydroxylated 2,4-diphenyl-6-aryl pyridines. Bioorg Med Chem 2010, 18:3066-77.

34. Gomez-Monterrey I: New benzo[g]isoquinoline-5,10-diones and dihydrothieno [2,3-b]naphtho-4,9-dione derivatives Synthesis and biological evaluation as potential antitumoral agents. Bioorg Med Chem 2003, 11:3769-3775.

35. Valderrama JA, González MF, Pessoa-Mahana D, Tapia RA, Fillion H, Pautet F, Rodriguez JA, Theoduloz C, Schmeda-Hirschmann G: Studies on quinones. Part 41: synthesis and cytotoxicity of isoquinoline-containing polycyclic quinones. Bioorg Med Chem 2006, 14:5003-5011.

36. Kast RE, Scheuerle A, Wirtz CR, Karpel-Massler G: The Rationale of Targeting Neutrophils with Dapsone during Glioblastoma Treatment. Anticancer Agents Med Chem 2011, 11:756-761.

37. Bissinger EM, Heinke R, Spannhoff A, Eberlin A, Metzger E, Cura V, Hassenboehler P, Cavarelli J, Schüle R, Bedford MT, Sippl W, Jung M: Acyl derivatives of $p$-aminosulfonamides and dapsone as new inhibitors of 
the arginine methyltransferase hPRMT1. Bioorg Med Chem 2011, 19:3717-3731.

38. Ghorab MM, Radwan MAA, Taha NMH, Amin NE, Shehab MA, Faker IMI: Dapson in Heterocyclic Chemistry, Part I: Novel Synthesis of SulfoneBiscompounds for Antimicrobial and Antitumor Activities. Phosphorus, Sulfur, and Silicon and the Related Elements 2008, 183:2891-2905.

39. Ghorab MM, Amin NE, El Gaby MSA, Taha NMH, Shehab MA, Faker IMI: Dapson in Heterocyclic Chemistry, Part III: Synthesis, Antimicrobial, and Antitumor Activities of Some New Bisheterocyclic Compounds Containing Biologically Active Diphenylsulfone Moiety. Phosphorus, Sulfur, and Silicon and the Related Elements 2008, 183:2918-2928.

40. Ghorab MM, Amin NE, El Gaby MSA, Taha NMH, Shehab MA, Faker IMI: Dapson in Heterocyclic Chemistry, Part IV: Synthesis of Some Novel Diphenylsulfones Containing Acetamide, Pyrrolidine, Piperazine, and Thiomorpholine Moieties as Antimicrobial and Antitumor Agents. Phosphorus, Sulfur, and Silicon and the Related Elements 2008, 183:2929-2942.

41. Ghorab MM, Radwan MAA, Taha NMH, Amin NE, Shehab MA, Faker IMI: Dapson in Heterocyclic Chemistry, Part II: Antimicrobial and Antitumor Activities of Some Novel SulfoneBiscompounds Containing Biologically Active Thioureido, Carbamothioate, Quinazoline, Imidazolidine, and Thiazole Moieties. Phosphorus, Sulfur, and Silicon and the Related Elements 2008, 183:2906-2917.

42. Al-Said MS, Bashandy MS, Al-Qasoumi SI, Ghorab MM: Anti-breast cancer activity of some novel 1,2-dihydropyridine, thiophene and thiazole derivatives. Eur J Med Chem 2011, 46:137-41.

43. Vojtek $A B$, Der $C J$ : Increasing complexity of the Rassignaling pathway. The Journal of Biological Chemistry 1998, 273:19925-19928.

44. Liang PH, Ko TP, Wang AHJ: Structure, mechanism and function of prenyltransferases. The Federation of European Biochemical Societies Journal 2002, 269:3339-3354.

45. Long SB, Casey PJ, Beese LS: Reaction path of protein farnesyltransferase at atomic resolution. Nat 2002, 419:645-650.

46. Mazieres J, Pradines A, Favre G: Perspectives on farnesyltransferase inhibitors in cancer therapy. Cancer Lett 2004, 206:159-167.

47. Hunt JT, Lee VG, Leftheris K, Seizinger B, Carboni J, Mabus J, Ricca C, Yan N, Manne V: Potent, cell active, non-thioltetrapeptide inhibitors of farnesyltransferase. J Med Chem 1996, 39:353-358.

48. Adjei AA: Farnesyltransferase inhibitors. Cancer Chemother Biol Response Modif 2001, 3:161-162.

49. Crul M, De Klerk GJ, Beijnen JH, Schellens JH: Ras biochemistry and farnesyltransferase inhibitors: a literature survey. Anticancer drugs 2001, 12:163-184

50. Scorilas A, Black MH, Talieri M, Diamandis EP: Genomic organization, physical mapping, and expression analysis of the human protein arginine methyltransferase 1 gene. Biochem Biophys Res Commun 2000, 260:466-474.

51. Cheung N, Chan LC, Thompson A, Cleary ML, So CWE: Protein argininemethyltransferase-dependent oncogenesis. Nat Cell Biol 2007, 9:1208-1215.

52. Skehan P, Storeng R, Scudiero D, Monks A, McMahon J, Vistica D, Warren JT, Bokesch H, Kenney S, Boyd MR: New colorimetric cytotoxicity assay for anticancer-drug screening. J Natl Cancer Inst 1990, 82:1107-1112.

\section{doi:10.1186/1752-153X-6-64}

Cite this article as: Al-Said et al.: Dapson in heterocyclic chemistry, part VIII: synthesis, molecular docking and anticancer activity of some nove sulfonylbiscompounds carrying biologically active 1,3-dihydropyridine, chromene and chromenopyridine moieties. Chemistry Central Journal 2012 6:64.

Publish with ChemistryCentral and every
scientist can read your work free of charge
"Open access provides opportunities to our
colleagues in other parts of the globe, by allowing
anyone to view the content free of charge."
W. Jeffery Hurst, The Hershey Company.
- available free of charge to the entire scientific community
- peer reviewed and published immediately upon acceptance
- cited in PubMed and archived on PubMed Central
- yours - you keep the copyright
submit your manuscript here:
http://www.chemistrycentral.com/manuscript/

